\title{
Global survey on COVID-19 beliefs, behaviors, and norms
}

\author{
Avinash Collis $^{\mathrm{a}, \mathrm{b}, 1}$, Kiran Garimella ${ }^{\mathrm{c}, 1}$, Alex Moehring ${ }^{\mathrm{d}, 1}$, M. Amin Rahimian ${ }^{\mathrm{a}, \mathrm{c}, \mathrm{e}, 1}$, \\ Stella Babalola ${ }^{\mathrm{f}, \mathrm{h}, 2}$, Nina Gobat ${ }^{\mathrm{i}, \mathrm{j}, 2}$, Dominick Shattuck ${ }^{\mathrm{h}, \mathrm{k}, 2}$, Jeni Stolow ${ }^{\mathrm{l}, \mathrm{j}, 2}$, Sinan Aral $^{\mathrm{a}, \mathrm{c}, \mathrm{d}, 3}$ \\ and Dean Eckles ${ }^{a, c, d, 3}$ \\ a Initiative on the Digital Economy, Massachusetts Institute of Technology \\ ${ }^{b}$ McCombs School of Business, The University of Texas at Austin \\ c Institute for Data, Systems, and Society, Massachusetts Institute of Technology \\ d Sloan School of Management, Massachusetts Institute of Technology \\ e Department of Industrial Engineering, University of Pittsburgh \\ ${ }^{\dagger}$ Bloomberg School of Public Health, John Hopkins University \\ h John Hopkins Center for Communications Programming \\ i University of Oxford \\ j Global Outbreak Alert and Response Network \\ k Breakthrough ACTION \\ ' College of Public Health, Temple University \\ ${ }^{1}$ Authors listed in alphabetical order. \\ ${ }^{2}$ Authors listed in alphabetical order. \\ 3 To whom correspondence should be addressed: mit covid survey researchers@mit.edu, \\ eckles@mit.edu, sinan@mit.edu.
}

\section{Abstract}

Policy and communication responses to COVID-19 can benefit from better understanding of people's baseline and resulting beliefs, behaviors, and norms. From July 2020 to March 2021 , we fielded a global survey on these topics in 67 countries yielding over 2.0 million responses. This paper provides an overview of the motivation behind the survey design, details the sampling and weighting designed to make the results representative of populations of interest and presents some insights learned from the survey. Several studies have already used the survey data to analyze risk perception, attitudes towards mask wearing and other preventative behaviors, as well as trust in information sources across communities worldwide. This reso urce can open new areas of inquiry in public health, communication, and economic policy by leveraging one of the first ever large-scale, rich survey data on beliefs, behaviors, and norms during a global pandemic in new and innovative ways. 


\section{Introduction}

Nonpharmaceutical interventions (NPIs) in response to COVID-19 often depend for their effectiveness on the behavioral responses of the public. Even with a vaccine, uptake is not entirely in the control of experts and policy-makers. Rather than being a small factor, there is growing evidence that people's prevention behaviors are dramatically influenced by many social and cultural factors (Ferguson 2007, West et al. 2020, Van Baven et al. 2020). Analyses of mobility data reveal that people's movement is predicted and perhaps caused by their partisan affiliation (Allcott et al. 2020), media consumption (Bursztyn et al. 2020), and the behaviors of their social networks (Bailey et al. 2020). Thus, the epidemiological and economic effects of policies that close (or open) businesses and schools are substantially determined by people's beliefs. This is consistent with the recognition, at least among public health experts, that health communication is a core part of effective response to epidemics, ideally in concert with other policies and interventions. However, developing and deploying effective policies and communication strategies demands data about people's beliefs and how they have been affected by prior exposure to information from governments, peers, and media - and this data is largely lacking, even as massive troves of medical and behavioral traces are used by researchers (Van Bavel et al. 2020).

Survey data can help fill this gap in our understanding of individuals' beliefs, behaviors, and perceptions. This motivated us to conduct a large-scale, international survey related to COVID19 in over 60 countries to help policymakers and researchers better mo nitor and understand people's knowledge, beliefs, behaviors, and risk perceptions across the world through a collaboration with Facebook and Johns Hopkins University, and with input from experts at the World Health Organization and the Global Outbreak Alert and Response Network. The survey's purpose was to guide policy and research around individual responses to COVID-19 beyond symptoms and the most closely associated behaviors. The survey ran from July 2020 until March 2021. It was translated to 51 languages and fielded in 67 countries, yielding over 2.0 million responses. The full survey instrument is provided in Appendix C.

The survey is organized into blocks based on the question topics. Every survey begins with questions from the same five blocks: information exposure, knowledge, vaccine and healthcare, and demographics. In snapshot countries, all respondents are shown an information block and then three additional blocks that are randomly selected from the remaining blocks. In multi-wave countries, respondents are shown four randomly selected blocks. Precise questions and the codebook for the data can be found in the appendix. In constructing the survey instrument, we drew on input from a wide set of domain experts. The survey consisted of questions related to COVID-19 information exposure and trust in information sources, knowledge about the virus, community norms, prevention behaviors, beliefs about efficacy of measures, vaccine acceptance, risk perceptions and locus of control in addition to demographics. The survey data includes weights that use the rich information Facebook has about its users to reduce bias from non-response and differential Facebook use among different subpopulations. 


\section{Methods}

\section{Sampling \& weighting}

The survey was fielded in two different ways. First, in countries with a sufficient pool of users to sample, we fielded a multi-wave survey that ran continuo usly in multiple two-week waves from September 2020 until February, 2021. In each wave, Facebook aimed to deliver 3,000 respondents to our survey. In countries with a more limited survey pool, we fielded a snapshot survey where Facebook aimed to deliver 3,000 respondents over a two week period; this was done twice, first in July, 2020 and then in November, 2020.

The Facebook team uses non-response modeling and poststratification techniques from survey statistics to design the two following components (Barkay et al. 2020, Groves and Lyberg, 2010):

1. Sampling - deciding who to present with the invitation to participate in the survey.

2. Weighting - providing a weight per user so that respondents better represent the target population as a whole.

Using the total survey error framework, the goal of the sampling and weighting steps are to minimise the representation error due to the coverage, sampling variability, and nonresponse biases.

\section{Completion types and associated analytical samples}

The MIT team supplied binary survey completion flags (binary indicators of whether or not each respondent has completed the survey) along with a respondent identifier (a random number associated with each survey respondent) back to the Facebook team. No other data about respondents was sent by MIT to Facebook. We provide the completion flags for the following two analytical samples:

1. Respondents who have completed the basic knowledge and demographics parts of the survey. This part consists of a briefing followed by questions about information exposure, availability of treatments and vaccines, and contact with healthcare workers, as well as gender, age, education, overall health, country, and in the case of the US and India, state as well. We call this the demographic completion type.

2. Respondents who have reached the end of the entire survey, viewing (and typically answering) additional questions about information sources; information needs; their knowledge about high risk populations, methods of transmission, and disease symptoms; norms and beliefs about distancing, mask wearing, and other preventative measures; risk perception and locus of control; work, travel and intentions to visit various locations, followed by a debrief. We call this the full survey completion type, though note that there can still be missing data due to no nresponse to individual questions.

Subsequently, the Facebook team computed and returned sets of survey weights to the MIT team, one set for each analytical sample. No other data about respondents was sent by 
Facebook to MIT besides a respondent identifier (a random number associated with each survey repondant), their language preference, these survey weights, and an indicator of whether these survey weights were clipped (see below).

\section{Non-response modeling and post-stratification}

Based on the various attributes of each client and their patterns of engagement with the Facebook platform, the Facebook team designed a regularized regression and post-stratification model to issue a set of weights for the survey respondents in each wave. Subsequently, the survey respondents are modeled as differing not only in their demographics (through poststratification), but also in their response or nonresponse behaviors. The regression model outputs the probability of responding to the survey as a function of the sample attributes. After training the regression model, the resultant non-response weights are calculated for each survey respondent as the inverse of their response probability (i.e., the inverse probability of sampling weights, IPSW). The Facebook team tested multiple models of survey nonresponse behavior with varying degrees of complexity (in terms of the number of included attributes and their interactions) and chose the most parsimonious model with low design effects (Roderick and Vartivarian, 2005). The non-response weights provide us with a representative sample of the Facebook active user base, irrespective of their non-response behavior or factors that might have prevented them from being exposed to Facebook's invitation to the survey. After weighting for nonresponse, by comparing the demographic data (age bracket and gender) of the respondents with the census data in each country, the weights were readjusted to match the target population which we aim to represent. This uses post-stratification, a common method in survey analysis to correct for known differences between the sample and target populations (Little 1993). Appendix A includes vario us consistency checks for representativeness of the weighted survey sample.

\section{Using the weights}

The weights are meant to be used in Hajek estimators (i.e., normalized importance sampling estimators) for measuring population means. Specifically, let $Y_{i}$ be an outcome variable of interest measured for the respondent $i$ whose weight is $w_{i}$. The Hajek estimator, $\hat{Y}$, for the population mean of the outcome, $\bar{Y}$, is given by:

$$
\hat{Y}=\frac{\sum_{i=1}^{n} w_{i} Y_{i}}{\sum_{i=1}^{n} w_{i}}
$$

This is the default in most statistical software for computing a weighted mean. Subsequently, if interested in population totals, analysts should use $N \hat{Y}$ as an estimator of the total outcome level where $N$ is the population size. That is, analysts should not use the weights in an unnormalized way, as in a Horvitz-Thompson estimator (i.e., an unnormalized importance sampling estimator), as, while the weights are approximately on the level of each country's adult population, the clipping and other adjustments to the weights make them unsuitable for direct estimation of total outcome levels without normalization. More generally, users can use these weights in other related estimators that appropriately normalize the weights (Särndal et al., 2003). 


\section{Results}

\section{Characteristics of the sample}

Figure 1 shows the sample size we obtained per country and the effective sample size (as measured by the Appendix Equation A.2). Though on average, we obtain 3,000 users per week, the effective sample size varies widely, with Bangladesh being only around 1,000 users.

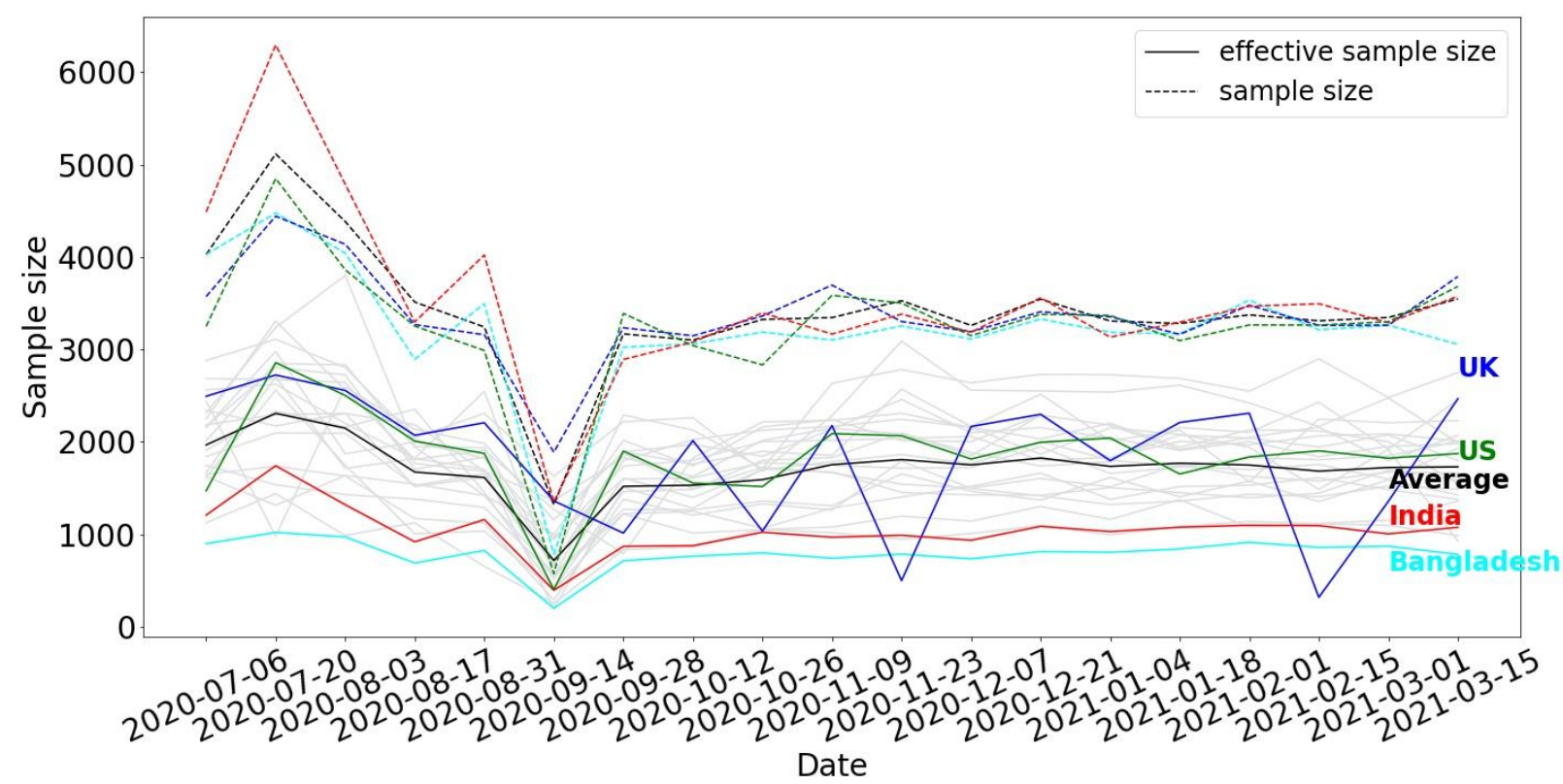

Figure 1: Sample size and effective sample size from our survey. We observe that we consistently sample

(except the wave starting on September 14, 2020) around 3,000 users every wave. However, the effective sample size varies widely among the countries and within each country. The $x$-axis indicates the start date of a two- week period (a 'wave') of data collection.

Next, we plot the conversion rate to the survey, i.e., how many users saw our survey prompt on their homepage, vs. how many clicked and completed our survey. We can see from Figure 2 that on average, we needed around 200 impressions for a single response. This is in line with the conversion for previous research using Facebook ads for surveys (Allcott et al., 2021). For most countries with good Facebook penetration (e.g. in Europe), the number is around 50. For some countries (e.g., Nigeria and India), the number was at least an order of magnitude higher. Our survey weights are designed to counter these biases in sampling. 


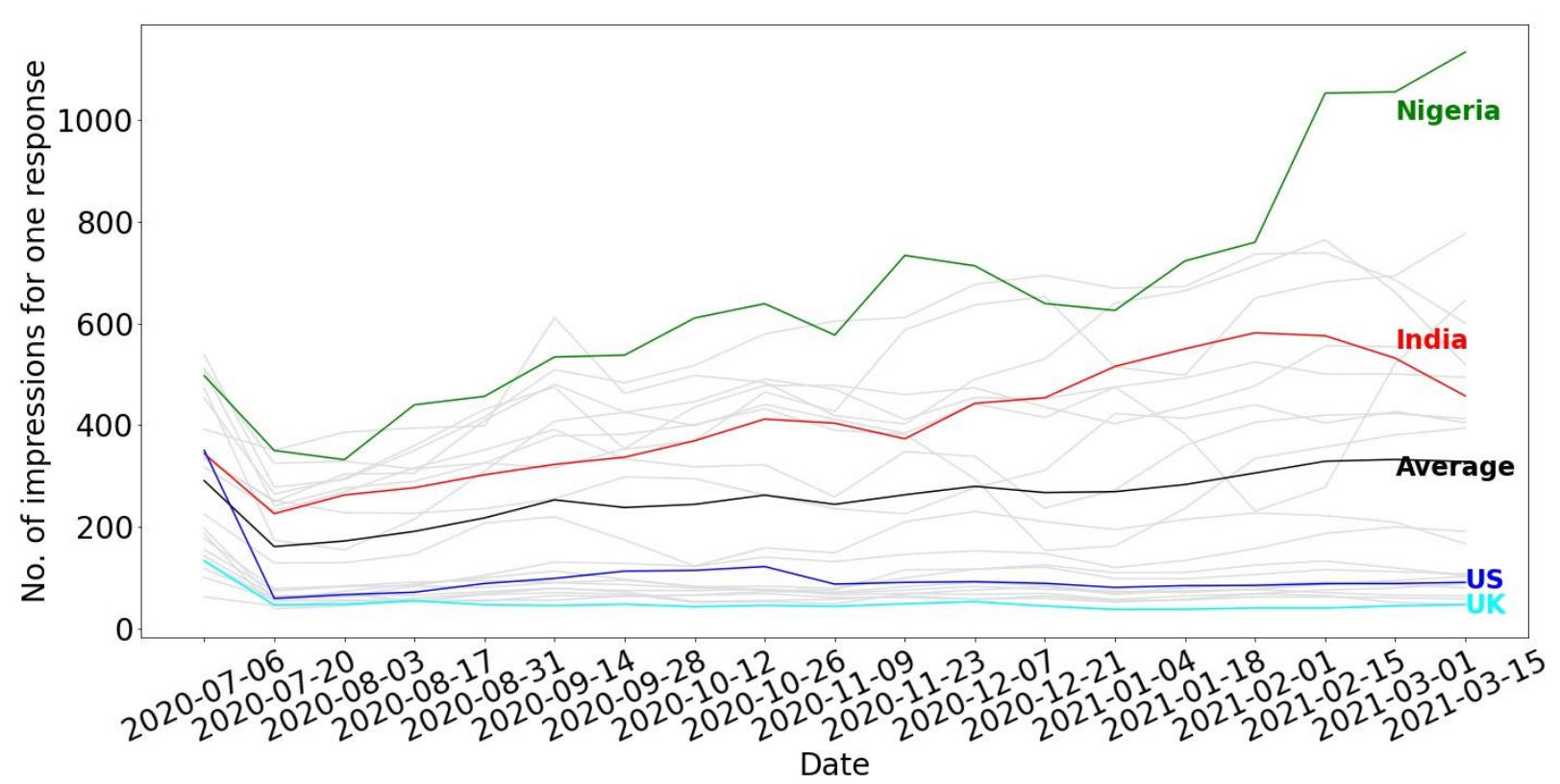

Figure 2: Number of impressions per response. We see the wide heterogeneity in the conversion across countries.

\section{Vaccine acceptance over time}

We look at vaccine hesitancy and its trends over time. First, we computed the fraction of respondents who say that they would take a vaccine or have taken the vaccine (starting July 2020). Figure 3 shows the trends for the 23 wave countries over the duration of the survey (July 2020 -- March 2021). For clarity, we highlighted 4 countries (Vietnam, US, Poland and Egypt) which have peculiar vaccine acceptance trends. From the figure, we observe a few clear trends: First, there is a huge heterogeneity across countries with Vietnam having a consistent vaccine acceptance of over $80 \%$ throughout the time period, whereas countries like the US and Poland experiencing an initial dip but improving in terms of acceptance later. Egypt has seen a steady decline in vaccine acceptance during the same period. On average, across the 23 wave countries, vaccine acceptance has been round the $65 \%$ mark and slightly improved since late 2020.

Starting in wave 9 (end of October 2020), we also asked the following question about vaccine norms: "Out of 100 people in your community, how many do you think would take a COVID-19 vaccine if it were made available?' The question helps us gauge perceptions of vaccine acceptance in the community. It is interesting to note that there is a significant difference between individual beliefs ("acceptance") and beliefs about others ("norms"). There is at least a $10 \%$ gap between them consistently. Respondents think that at least an additional $10 \%$ of the population would not take the vaccine, indicating the need for interventions that surface norms highlighting acceptance (Moehring et al., 2021). 


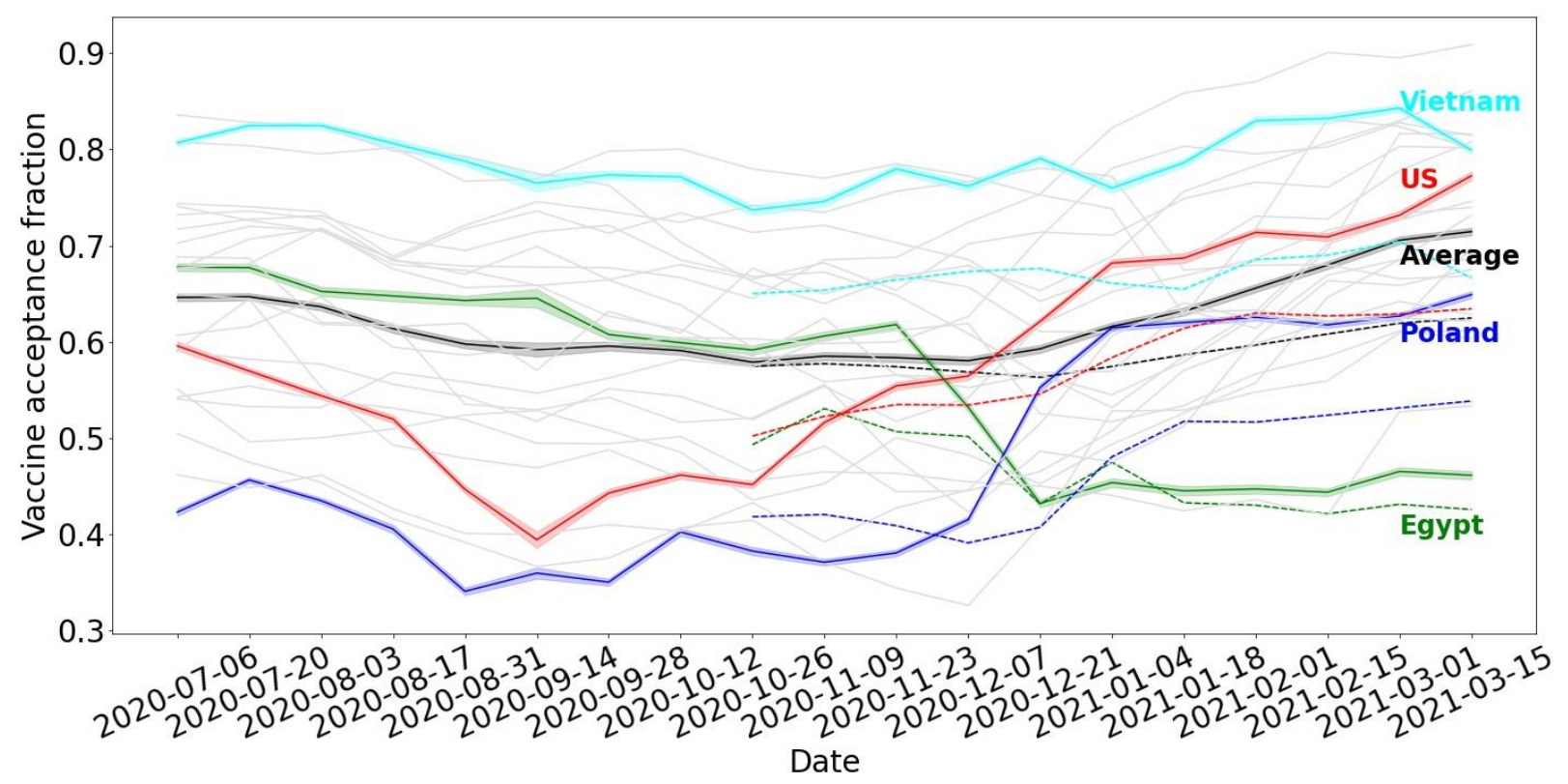

Figure 3: Vaccine acceptance over time

Figure 4 shows the fraction of responses to the vaccine acceptance question for the 4 countries. The figure shows the importance of the "Don't know" or people who are yet undecided on the vaccine. Consider the case of the US, where the fraction of users who say no to the vaccine has remained almost constant throughout the study period, whereas a large fraction of the unsure users changed their opinions in the later parts of 2020. Similarly, in Egypt, the fraction of users who oppose the vaccine as well as those who are unsure has increased in the past few months which is a good case study for policy intervention. Overall, on average, across the 23 countries in our dataset, vaccine acceptance or the fraction of users who are not sure has not changed since July 2020, which is indicative of the need for efforts in improving vaccine confidence.

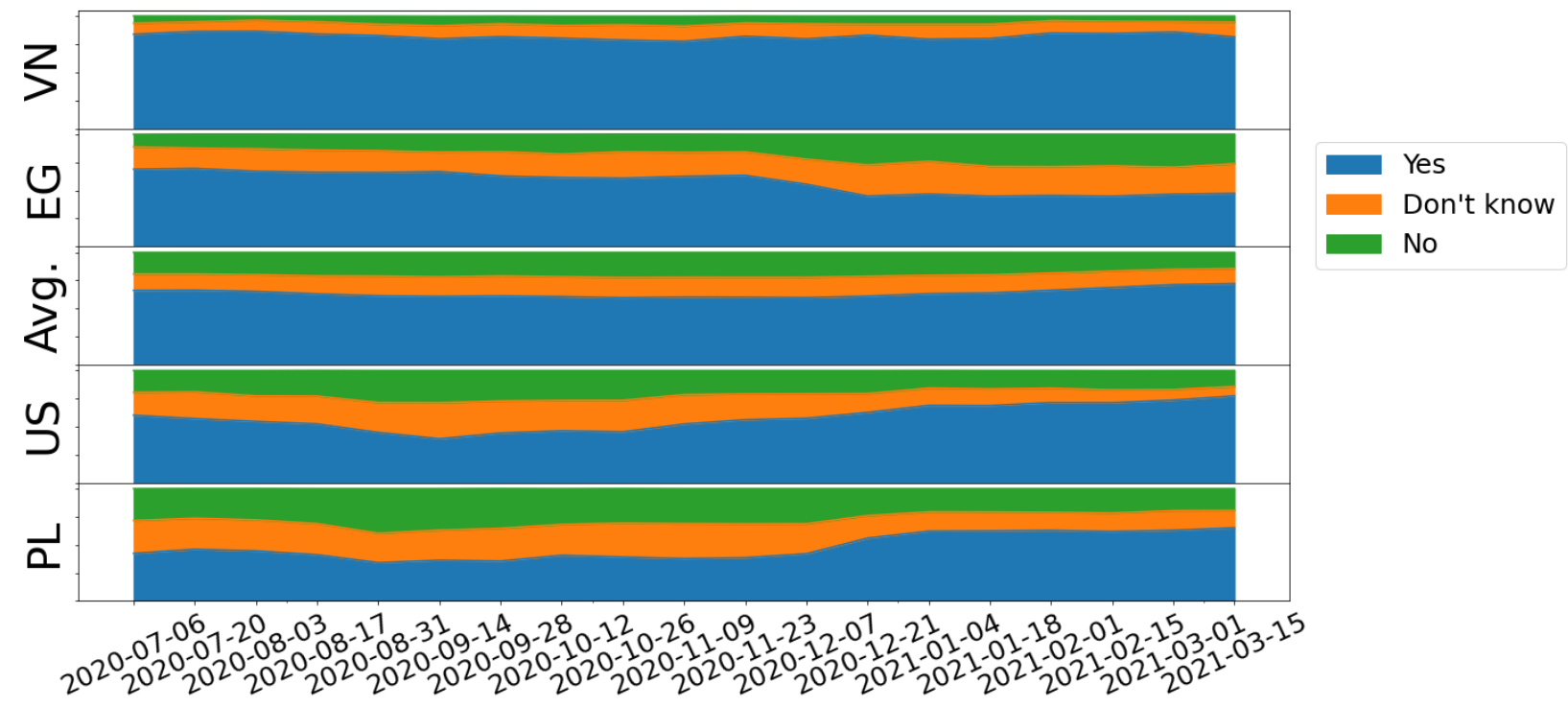


Figure 4: Distribution of responses for the vaccine acceptance question for 4 countries (along with the average across our sample). We can observe the wide variance across countries about the role of vaccine hesitant users (respondents saying "No") and unsure users (respondents saying "Don't know").

Next, we plotted the correlation between acceptance and norms for the 44 snapshot countries (for wave 9, in which the norms question was asked). We observe similar trends in Figure 5. In all the 44 countries, respondents think others are much less likely to get the vaccine than themselves. We highlighted 4 countries (CM: Cameroon, AZ: Azerbaijan, AF: Afghanistan, and KR: South Korea), which indicate the heterogeneity across countries.

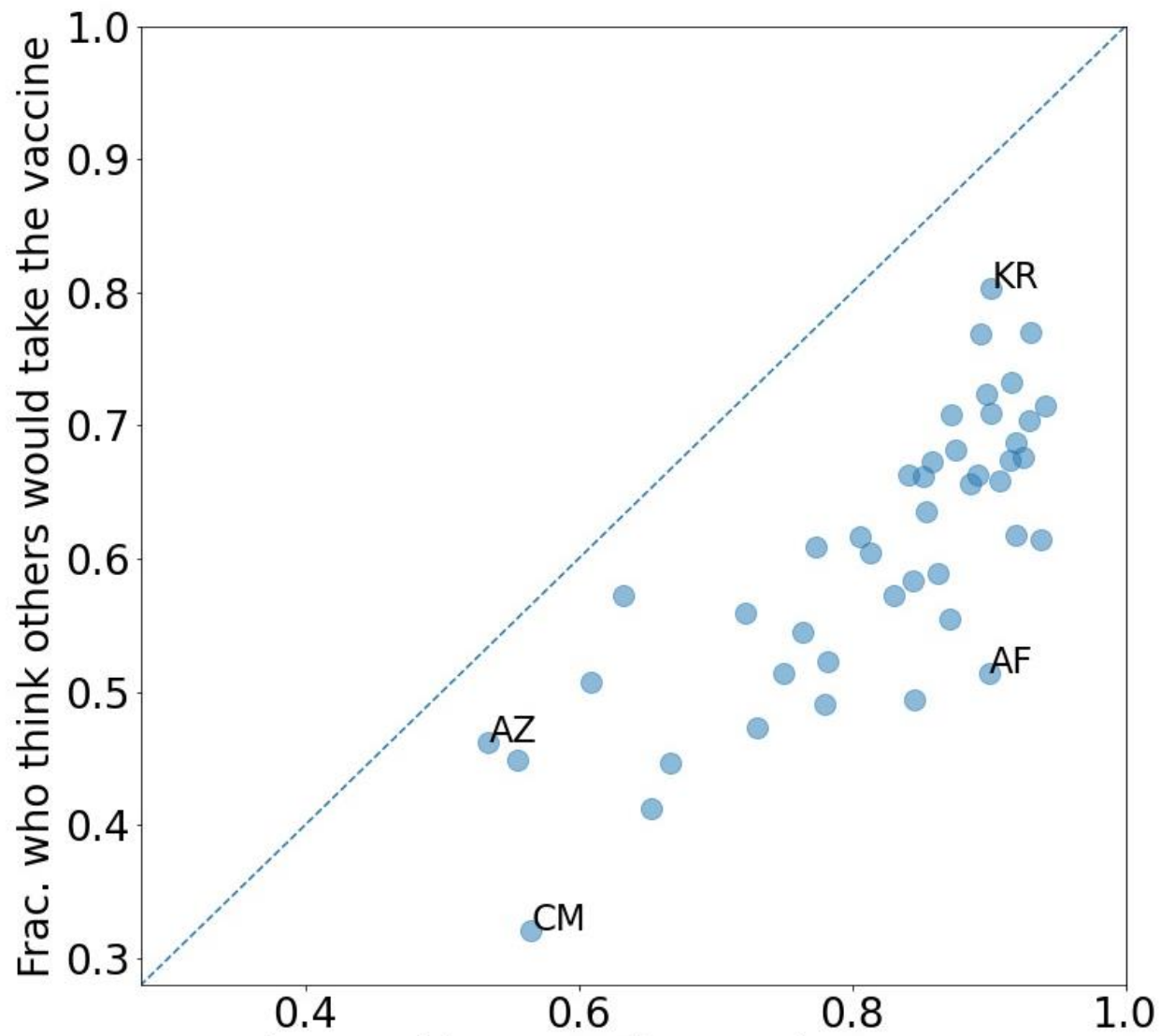

Frac. who would accept the vaccine or are unsure

Figure 5: Vaccine acceptance vs. vaccine norms. The scatter plot clearly shows that respondents think that far fewer of their fellow citizens would take the vaccine compared to the reality.

\section{Mismatch in COVID perceptions}

We asked two questions about the perception of seriousness of COVID and perceptions among the community.community_action_importance : "How important is it for you to take actions to prevent the spread of COVID-19 in your community?' (possible answers: Extremely important, Very important, Moderately important, Slightly important, Not important at all) and community_action_norms: "How important do other people in your community think it is to take actions to prevent the spread of COVID-19?'. If respondents themselves think of taking action 
against COVID to be extremely important, but think others do not take it seriously (or vice versa), they might adapt their behavior to take steps which would not be necessary. We see that there is a clear difference in the distributions across US and Japan, with most people in Japan having a congruent view, compared to the wide range of disagreement in the US.
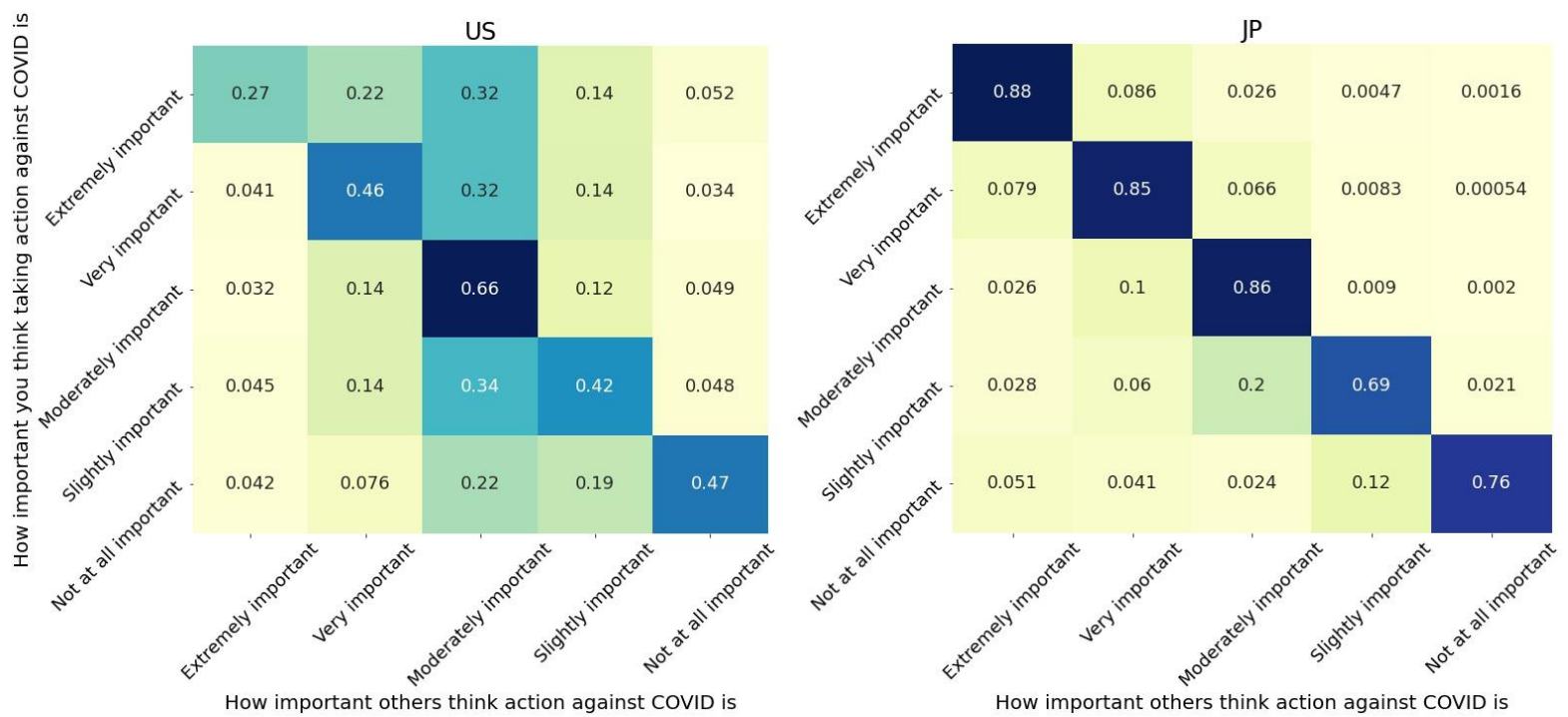

Figure 6: The figure shows the mismatch across two countries: the US and Japan. The $y$-axis indicates how serious the respondent considers COVID to be, and the $x$-axis indicates how serious they think others think COVID is.

\section{News sources and medium: Consumption vs. trust}

Finally, we asked for the sources/mediums users consumed COVID related information from, and their trust in these sources. Figure 7 shows the trends for consumption and trust for 5 sources: online news, radio, TV, local health workers and politicians. In a pandemic, an ideal situation for information consumption would be from a widely trusted source which is also widely consumed. However as we can see from the figure, most sources do not satisfy this criteria. Some interesting trends emerge: Politicians are the least trusted and in most countries the source of information. Television has high consumption but the trust varies widely among the countries in our sample. Local health workers are typically well trusted but they are not a source of information for most of the countries. This figure provides insights that the voices of local health workers, who are trusted, should be promoted more. 


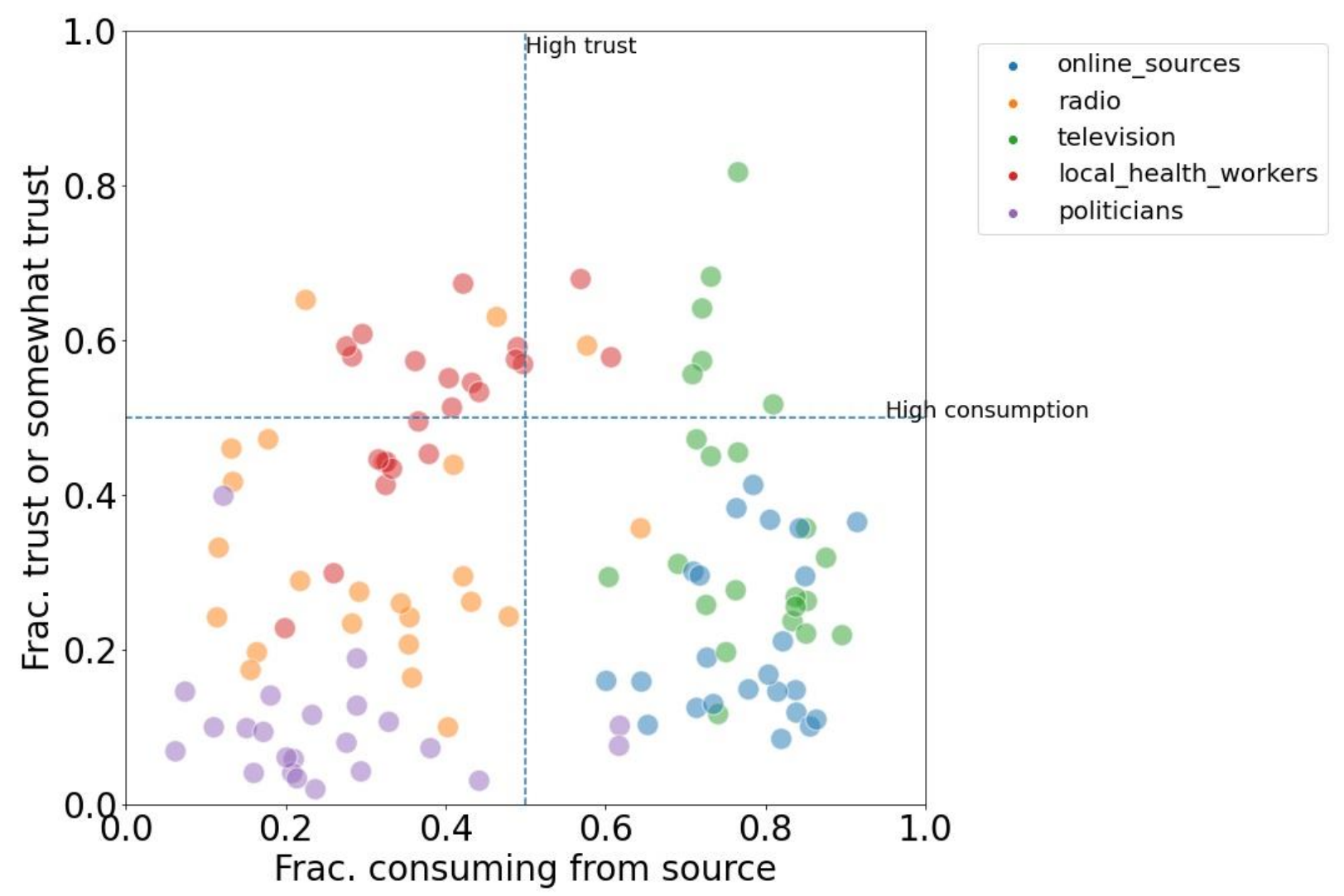

Figure 7: Trust vs consumption of news sources/mediums. The trust and consumption of various sources/mediums varies across countries.

\section{Discussion}

Several studies have used the COVID-19 beliefs, behaviors, and norms survey data to analyze risk perception, attitudes towards mask wearing and other preventative behaviors, as well as trust in information so urces across communities worldwide. Lu, Kong, and Shelley (2021) use the survey data to identify significant predictors of high risk perception in older adults and its association with their preventive behaviours and medical avoidance. They find accurate knowledge to be an important factor in disentangling this association. Joining the survey data with COVID-19 cases and death counts worldwide, Adjodah et al. (2021) show that mask wearing and attitudes towards masks are associated with fewer cases and deaths across different countries, controlling for socioeconomic factors such as population density, human development and mobility. Lu, Jin, and English's (2021) analysis of the survey data reveals that mask usage is higher in countries with more collectivistic (versus individualistic) cultures after controlling for a host of variables such as COVID-19 severity, government policy, population density, GDP per capita, and demographics. Other studies have used the survey measures such as vaccine acceptance directly as motivation or explanatory factors in their research (e.g., Matute et al. 2021). 
Moehring et al. (2021) use the randomized order of the survey questions to show that highlighting accurate information about vaccine norms increases vaccine acceptance. Several layers of randomization throughout the survey provide a ripe ground to explore priming, anchoring and information treatment effects on different demographics in a representative global sample (e.g., respondents are randomized to see questions about their risk perception and perceived control over health outcomes which affects their answers to follow up questions about their adherence to preventive measures in ways that can inform public health communication). The longitudinal data are collected over a period of global pandemic emergency that coincided with high profile events, providing natural experiment opportunities on national and international scales (e.g., the U.S. presidential election, epidemic peaks and emergency use approvals of vaccines in different countries, etc.) In addition to in-depth demographic, psychographic and sociometric measurements, the survey questions cover issues related to work, travel and the media. We expect the confluence of these factors will open new areas of inquiry in public health, communication, and economic policy and we are optimistic that future researchers will leverage this large-scale, rich survey data on beliefs, behaviors, and norms during the COVID-19 pandemic in new and innovative ways.

\section{Data Availability}

Aggregate data can be found here, and researchers can request access to respondent-level responses (microdata) by requesting access here.

\section{References}

Adjodah, Dhaval, et al. "Association between COVID-19 outcomes and mask mandates, adherence, and attitudes." PLOS ONE 16.6 (2021): e0252315.

Allcott, Hunt, Levi Boxell, Jacob Conway, Matthew Gentzkow, Michael Thaler, and David Yang. "Polarization and public health: Partisan differences in social distancing during the coronavirus pandemic." Journal of Public Economics 191 (2020): 104254.

Allcott, Hunt, Matthew Gentzkow, and Lena Song. Digital addiction. No. w28936. National Bureau of Economic Research, 2021.

Bailey, Michael, Drew M. Johnston, Martin Koenen, Theresa Kuchler, Dominic Russel, and Johannes Stroebel. Social networks shape beliefs and behavior: evidence from social distancing during the Covid-19 pandemic. No. w28234. National Bureau of Economic Research, 2020.

Barkay, Neta, Curtiss Cobb, Roee Eilat, Tal Galili, Daniel Haimovich, Sarah LaRocca, Katherine Morris, and Tal Sarig. 2020. "Weights and Methodology Brief for the COVID19 Symptom Survey by University of Maryland and Carnegie Mellon University, in Partnership with Facebook." arXiv. http://arxiv.org/abs/2009.14675. 
Bursztyn, Leonardo, Aakaash Rao, Christopher P. Roth, and David H. Yanagizawa-Drott. Misinformation during a pandemic. No. w27417. National Bureau of Economic Research, 2020.

Ferguson, Neil. "Capturing human behaviour." Nature 446, no. 7137 (2007): 733-733.

Groves, Robert M., and Lars Lyberg. "Total survey error: Past, present, and future." Public opinion quarterly 74.5 (2010): 849-879.

Little, Roderick J. "Post-stratification: A modeler's perspective." Journal of the American Statistical Association 88.423 (1993): 1001-1012.

Little, Roderick J., and Sonya Vartivarian. "Does weighting for nonresponse increase the variance of survey means?." Survey Methodology 31.2 (2005): 161-168.

Lu, Jackson G., Peter Jin, and Alexander S. English. "Collectivism predicts mask use during COVID-19." Proceedings of the National Academy of Sciences 118.23 (2021).

Lu, Peiyi, Dexia Kong, and Mack Shelley. "Risk Perception, preventive behavior, and medical care avoidance among American older adults during the COVID-19 pandemic." Journal of Aging and Health (2021): 08982643211002084.

Matute, Jorge, et al. "Are you getting it? Integrating theories to explain intentions to get vaccinated against COVID-19 in Spain." Journal of Risk Research (2021): 1-20.

Moehring, Alex, Avinash Collis, Kiran Garimella, M. Amin Rahimian, Sinan Aral, and Dean

Eckles. "Surfacing norms to increase vaccine acceptance." Available at SSRN 3782082 (2021).

Särndal, Carl-Erik, Bengt Swensson, and Jan Wretman. Model Assisted Survey Sampling. Springer Science \& Business Media, 2003.

Van Bavel, Jay J., Katherine Baicker, Paulo S. Boggio, Valerio Capraro, Aleksandra Cichocka, Mina Cikara, Molly J. Crockett et al. "Using social and behavioural science to support COVID-19 pandemic response." Nature human behaviour 4, no. 5 (2020): 460-471. West, Robert, Susan Michie, G. James Rubin, and Richard Amlôt. "Applying principles of behaviour change to reduce SARS-CoV-2 transmission." Nature human behaviour 4, no. 5 (2020): 451-459.

\section{Acknowledgements}

This work was funded in part by a grant from Facebook to the MIT Initiative on the Digital Economy. This survey was a collaborative effort involving contributions from individuals at multiple institutions, especially the Massachusetts Institute of Technology, Johns Hopkins University, the Global Outbreak Alert and Response Network (GOARN), the World Health Organization, and Facebook. At Facebook, key contributors were Esther Kim, Kelsey Mulcahy, Praveen Raja, Stephanie Sasser, Carlos Velasco, and Thomas Wynter. At MIT, we thank Aileen Ruel and Susan Watts for managing other institutions gaining access to the microdata. 


\section{Appendix A: Survey weights}

For each country, the Facebook team considered a two-way cross-classification of the age brackets and gender, and provided weights for the country's adult population. Although poststratification reduces the bias and increases the representativeness of the survey sample, the added adjustment to the weights induces an increased design effect. We aimed to keep the overall design effects due to both the non-response and post-stratification weighting below four, and it remained below two for most counties (Appendix Figure 1).

Appendix Figure 1. Design effect from weighting wave 1, using the demographic completion weights, for two of the questions that occur prior to the demographics block. Design effects for most countries are near or below two.

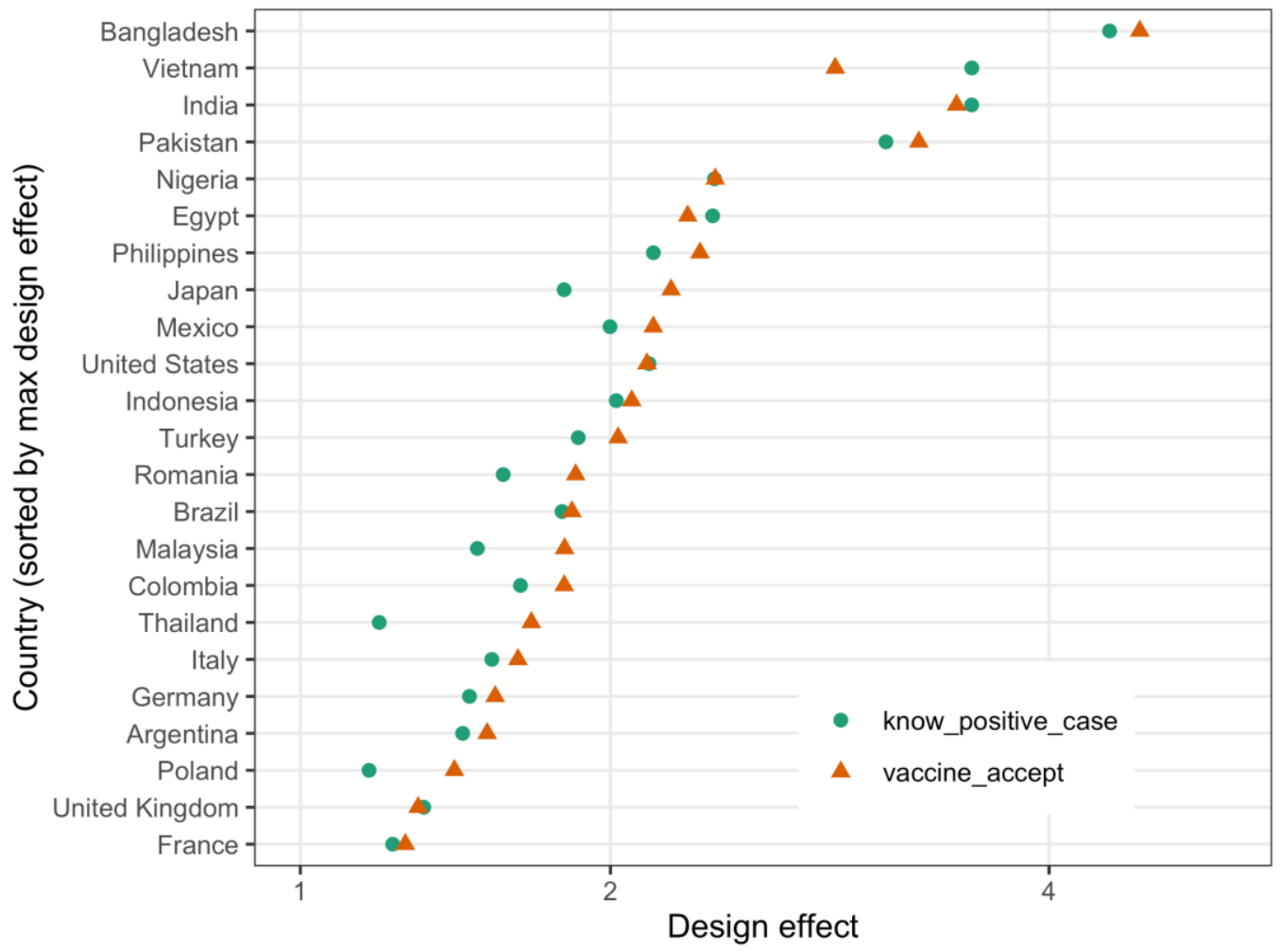

Using the survey data, we can plot the self-reported demographics of each country with and without the weights to see the effect of post stratification (Appendix Figure 2). In some cases, Facebook's inferred demographics may not match what the users report in the survey. This causes a mismatch between the census data and weighted self-reported demographics (e.g., female population in Pakistan, India and Bangladesh in Appendix Figure 2 remain below their census levels). Since our survey results come with self-reported demographic information, the researchers who use this data can perform their own adjustments to the weight in trying to match their target population's demographics. 
Appendix Figure 2. Illustration of change in self-reported demographics from weighting of wave 1. Unweighted proportions in brown show country-specific biases. These are substantially reduced when using weights - here the weights for the demographic completion type. The selfreported fraction of females remains statistically significantly below 0.5 in Pakistan, India, and Bangladesh even after weighting.
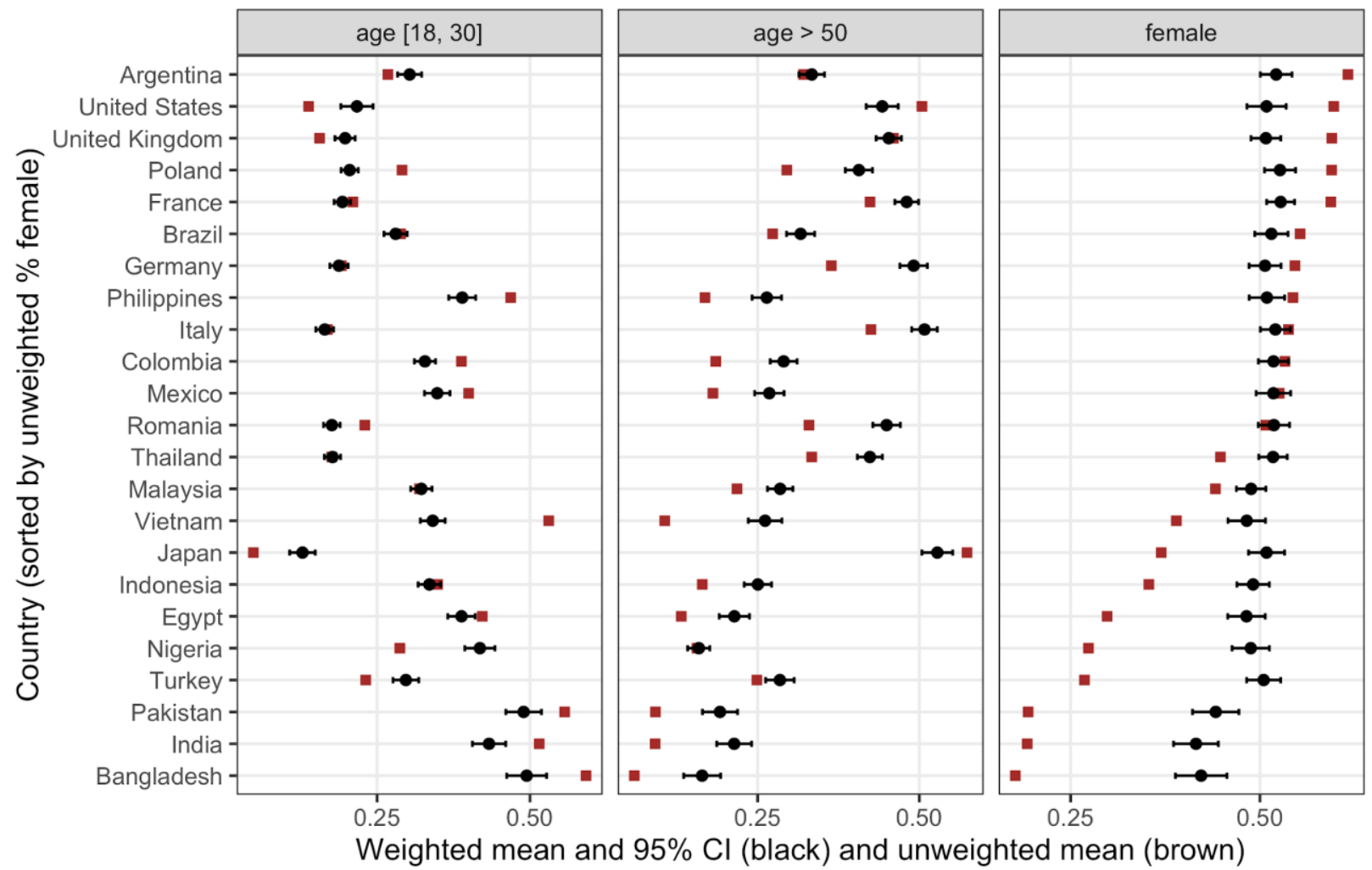


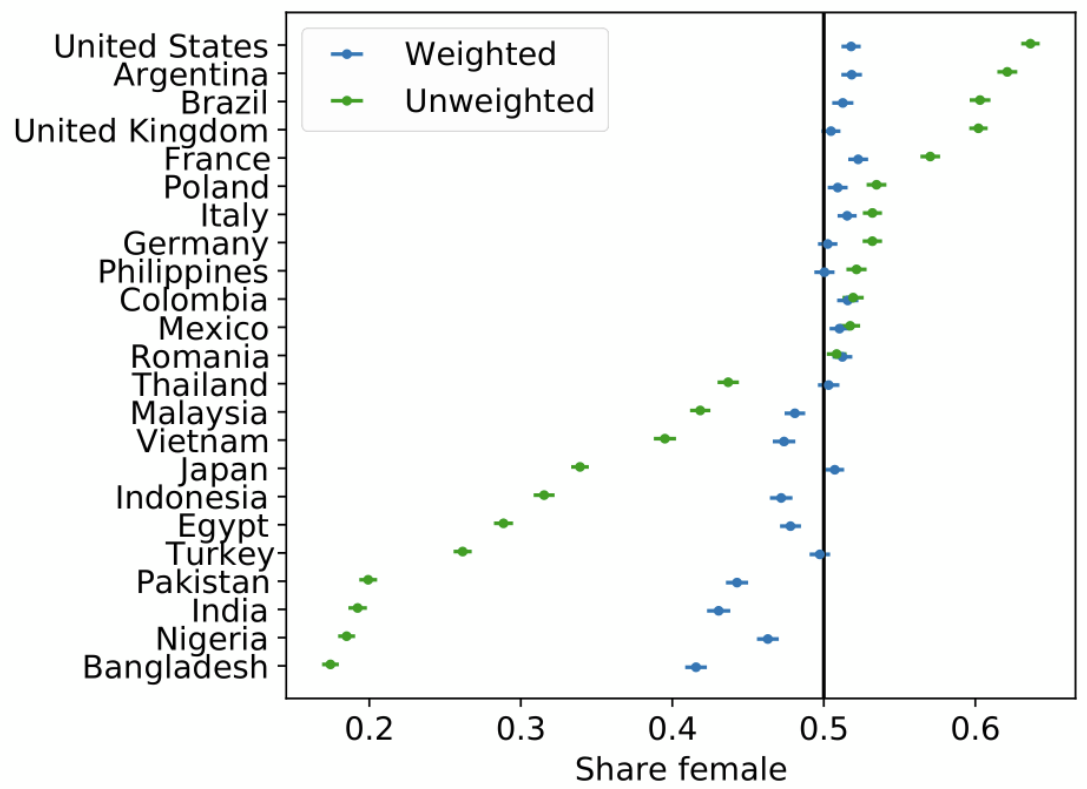

For some countries, weights for another target population are provided (in addition to the adult population weights). In particular, for some countries where data on their Internet-using population was available, there was (a) substantial divergence between their adult Internet users and adult population and (b) substantial divergence between the respondent population and the adult population, such that post-stratification by age and gender results in a large reduction in effective sample size and may also leave much bias remaining. For some of these countries, and where data on demographics of Internet users is available, we include weights where the target population is adult Internet users. Targeting the population of internet-using adults will reduce the design effect (Appendix Figure 3) as presumably the demographics of the Facebook active user base is more similar to that of adult Internet users than all adults. 
Appendix Figure 3. Design effect from weighting when post-stratifying to match adult population or Internet-using adult population, for those wave countries where the latter are available. Both design effects are computed for wave 1 with the demographic completion type for knowing a positive case.

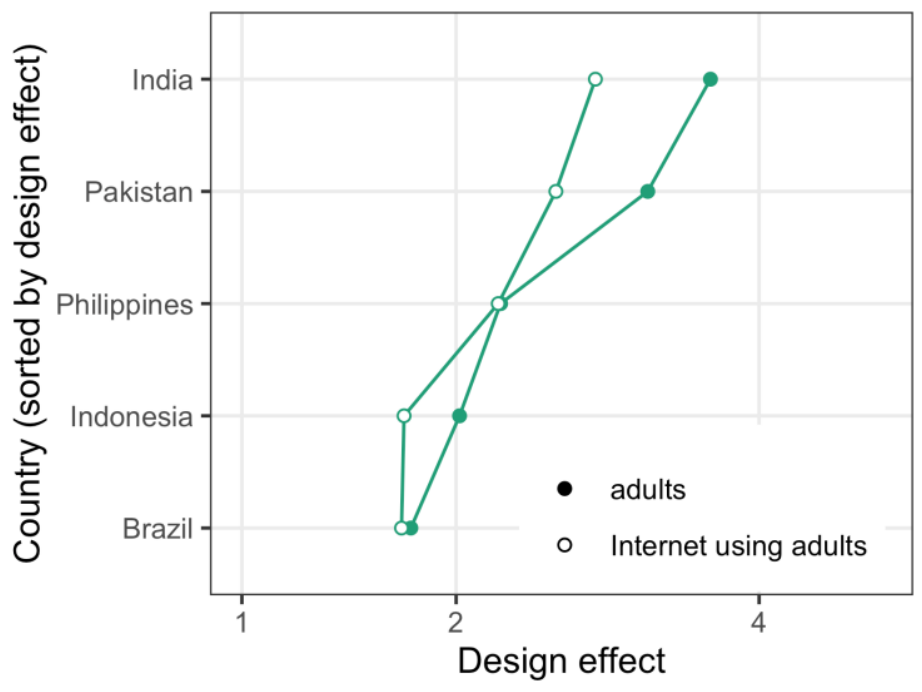

Finally, the weights were clipped (i.e., trimmed, Winsorized) from below and above to control their variance and especially to reduce sensitivity to a few samples with very large weights. In particular, Facebook trimmed the weights within each country at 10 times above and 30 times below their (untrimmed) means for that country; then, because of possible mismatch between the country associated with a respondent in Facebook data and that from the survey data, MIT made further adjustments to the weights within each country. Specifically, MIT has two ways of identifying each participant's country: (i) the respondent's self-reported country in the demographics part of the survey and (ii) the GeolP location data that MIT obtains from Qualtrics for each respondent. The Facebook team has their own way of determining each participant's country using their internal data which per our legal agreements is not shared with MIT. In addition to providing a weight for each survey repondant, the Facebook team also provides an indicator variable of whether the weight for that repondant has been clipped or not.

Subsequently, the MIT team compares each respondent's weight with other respondents that have the same reported or GeolP-identified country. If a participant's weight is larger than the level at which Facebook has clipped the weights for that country from above (inferred from the clipping indicators), then the MIT team determines that the participant's country is misidentified and such participants' weights are subsequently reset to zero (removed from the sample). Although such misidentified cases happen very rarely (a few such cases have been observed thus far), this correction is important because such large weights could substantially bias the survey results.

Given the final weight $w_{i}$ for each respondent $i$, we can compute the design effect and effective sample size. First, one can compute these without reference to a particular outcome: 


$$
\begin{aligned}
& \text { (Kish) design effect }=\frac{n \sum_{i=1}^{n}\left(w_{i}\right)^{2}}{\left(\sum_{i=1}^{n}=1\right.}, \\
& (\text { Kish }) \text { effective sample size }=\frac{\left(\sum_{i=1}^{n} w_{i}\right)^{2}}{\sum_{i=1}^{n}\left(w_{i}\right)^{2}},
\end{aligned}
$$

where $n$ is the total number of completed survey responses. These formulas were used by the Facebook team to benchmark the design effects as they did not have access to the outcome data (recall that only random respondent IDs and completion indicators were sent from MIT to Facebook). On the other hand, MIT can additionally compute design effects and effective sample sizes for each outcome, accounting for the correlation between the outcome and the weights. Then the design effect is estimated using the ratio between the variance of the weighted estimator and an unweighted estimator using an equality probability sample; this can also account for further changes in missingness for that particular item, which can be assumed to be missing unconditionally (completely) at random. This is what is reported in Appendix Figures 1 and 3.

\section{Internal consistency of the non-response models}

Because of the creation of weights for two different completion types, it is possible to test some observable implications of the assumptions of the non-response model (e.g., whether someone responds is as-good-as-random random conditional on the observables used in the nonresponse model above). In particular, for the questions in the initial blocks of the survey, estimation using either the demographic completion type or full survey completion type should yield very similar answers. Analysis of two substantive questions suggests that these observable implications of this assumption are not egregiously violated (Appendix Figure 4). 
Appendix Figure 4. Comparison of weighted estimates of proportions using the demographic completion and full survey completion weights for wave 1. Under conditional ignorability of nonresponse, these should be the same in large samples.
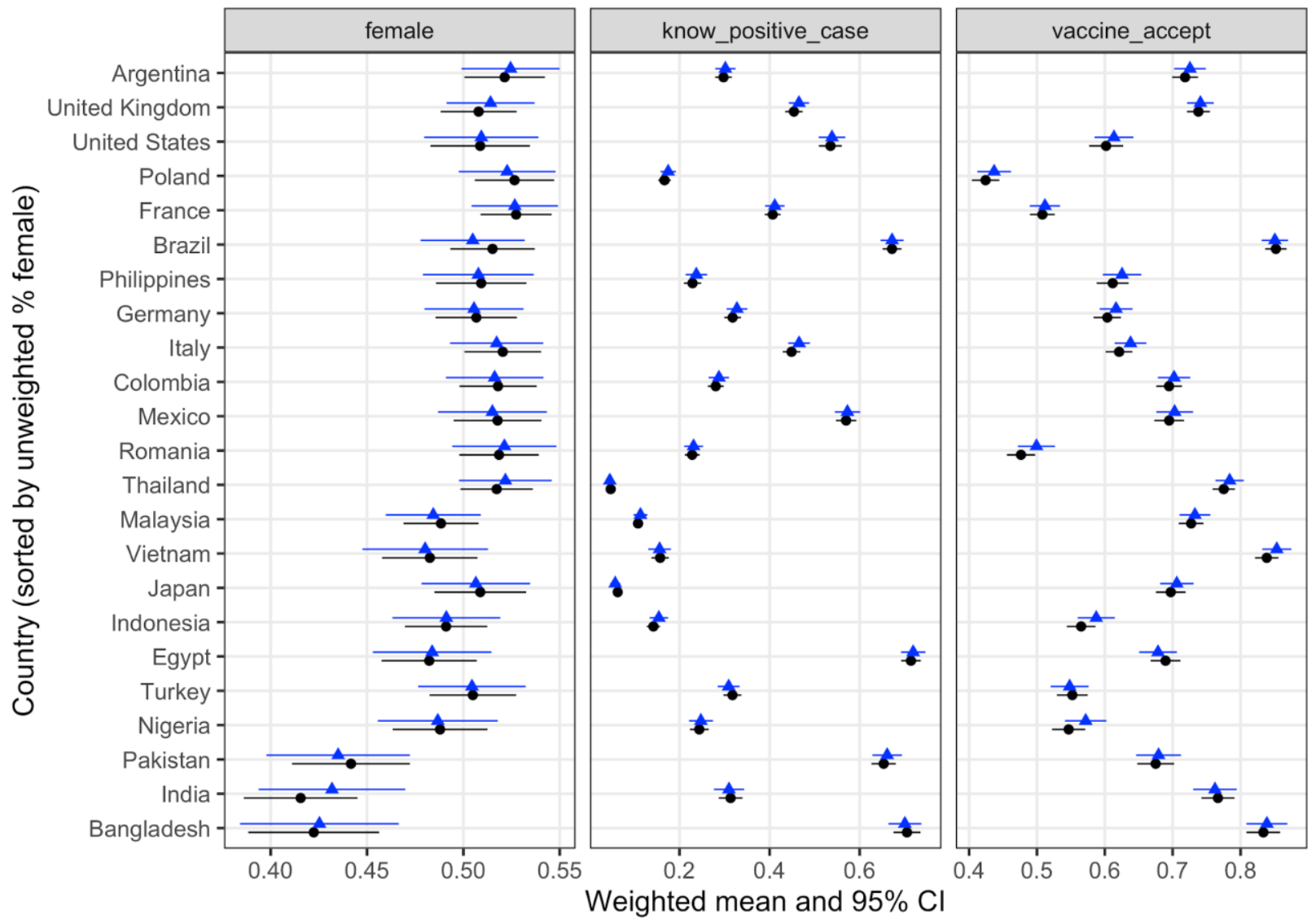

completion_type $\quad$ + demo $\uparrow$ full 


\section{Appendix B: Data Dictionary}

\section{Missing Values}

Data can be missing for a number of reasons, some missing is by design through rando mization and other is due to selective answering and dropoff. For all questions, missing values are coded as follows.

- NULL: Null values indicate the question was not shown either due to randomization or because the respondent left the survey before this point.

- -1 : The question was viewed but not answered and the respondent completed the block containing this question.

- $\quad-2$ : The question was viewed but not answered and the respondent did not complete the block containing this question.

\section{Sample Code}

Below are commands that can load the data in commonly used data analysis software. The data is a compressed tab-delimited text file.

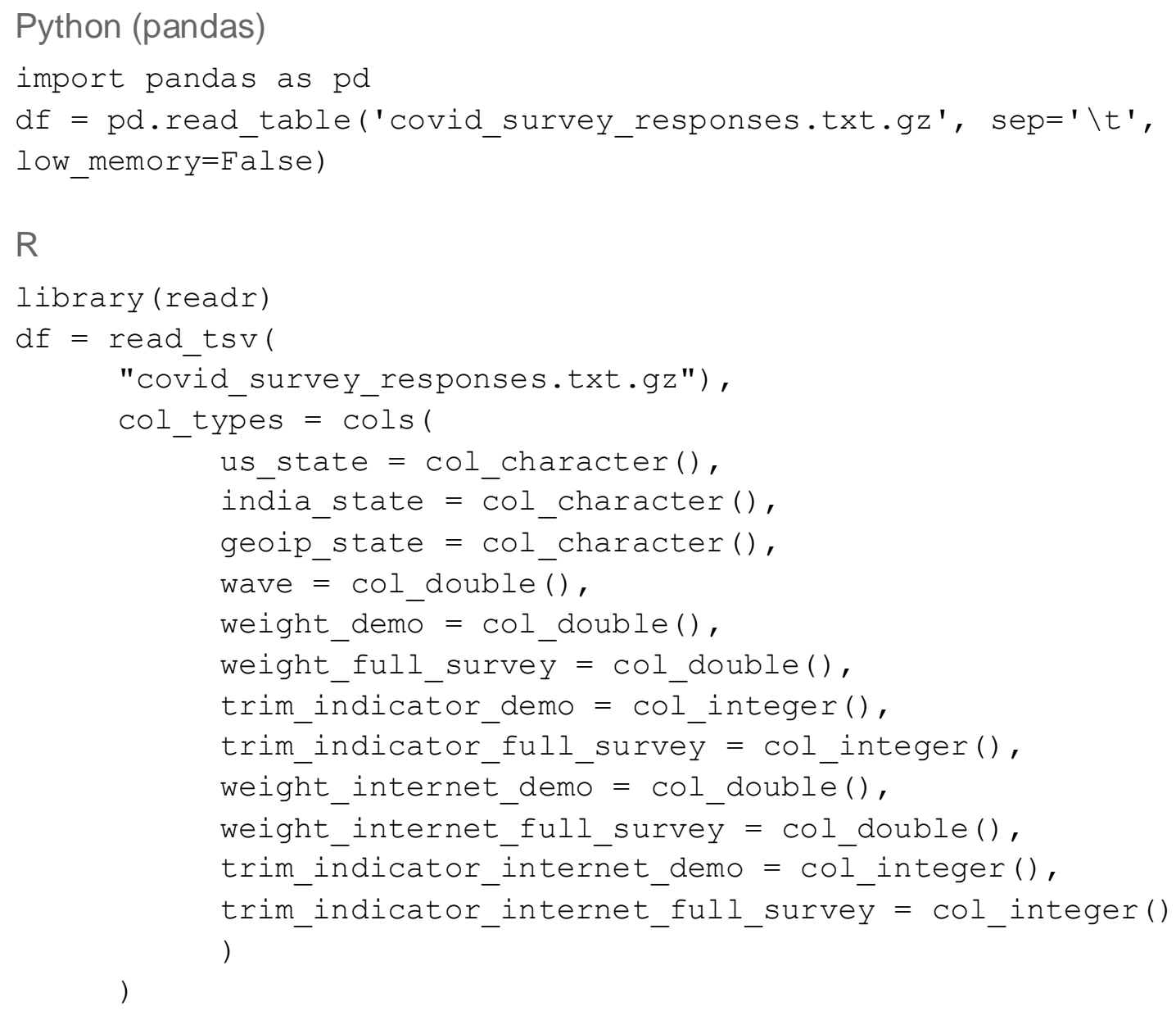




\section{Meta Data}

\begin{tabular}{|c|c|c|}
\hline Variable & Type & Description \\
\hline id & string & Unique id assigned to a survey response \\
\hline start_date & YYYY-MM-DD & Date survey was started \\
\hline duration & integer & Duration of survey in seconds \\
\hline language & string & Language code survey was taken in \\
\hline country & string & Inferred country of residence (See notes below) \\
\hline reported_country & string & $\begin{array}{l}\text { Self-reported country of residence (See notes } \\
\text { below) }\end{array}$ \\
\hline geoip_country & string & $\begin{array}{l}\text { Country of residence inferred from Qualtrics GeolP } \\
\text { (See notes below) }\end{array}$ \\
\hline progress & integer & $\begin{array}{l}\text { Integer representing share of survey complete, } \\
\text { between } 0 \text { and } 100 .\end{array}$ \\
\hline survey_type & string & Survey version, either waves or snapshot \\
\hline survey_version & string & $\begin{array}{l}\text { One of } v 1, v 2, v 3 \text {, representing the iteration of the } \\
\text { survey taken. }\end{array}$ \\
\hline wave & int & $\begin{array}{l}\text { Wave number if survey_type is waves, null } \\
\text { otherwise }\end{array}$ \\
\hline device_type & string & $\begin{array}{l}\text { Device used to complete survey (desktop, mobile, } \\
\text { other) }\end{array}$ \\
\hline browser & string & Name of browser used to complete survey \\
\hline demo_complete & bool & $\begin{array}{l}\text { Indicator if the respondent answered all } \\
\text { demographic questions not including location } \\
\text { questions and had a non-null country }\end{array}$ \\
\hline finished & bool & $\begin{array}{l}\text { Indicator if the respondent reached the end of the } \\
\text { survey }\end{array}$ \\
\hline $\begin{array}{l}\text { weight_[ [complet } \\
\text { ion type] ] }\end{array}$ & float & $\begin{array}{l}\text { Weight using respondents who complete } \\
{[[\text { completion type]] as an analytical sample and adult }} \\
\text { population as a target population }\end{array}$ \\
\hline $\begin{array}{l}\text { weight_internet_- } \\
\text { [ [ completion } \\
\text { type] ] }\end{array}$ & float & $\begin{array}{l}\text { Weight using respondents who complete } \\
{[[\text { completion type]] as an analytical sample and adult }} \\
\text { internet users as a target population }\end{array}$ \\
\hline
\end{tabular}




\begin{tabular}{|c|c|c|}
\hline trim_indicator & bool & $\begin{array}{l}\text { Indicator if the respondent's weight was clipped by } \\
\text { Facebook. }\end{array}$ \\
\hline display_order & string & $\begin{array}{l}\text { JSON string containing the order questions, } \\
\text { answers, and blocks were displayed. } \\
\text { "block_order": \{ } \\
\text { block name: block order } \\
\text { \} "question_order": \{ } \\
\text { question id: question order within } \\
\text { block. Only populated for questions } \\
\text { that have a randomized order. } \\
\text { \} "block_completions": \{ } \\
\text { block: Dictionary indicating if } \\
\text { block was started and/or completed. } \\
\text { \} "last_finished_block": Last block } \\
\text { completed in the survey } \\
\text { "last_started_block": Last block } \\
\text { started in the survey } \\
\text { "answer_order": \{ } \\
\text { question: Dictionary mapping } \\
\text { question answers to display positions. } \\
\text { Only populated for questions with } \\
\text { randomization in answers. } \\
\text { \} } \\
\text { \} } \\
\text { Note for roughly } 600 \text { responses the display order } \\
\text { was improperly logged and will be null. For these } \\
\text { responses, we are also unable to provide the full } \\
\text { missingness codes. For these } 600 \text { responses, null } \\
\text { implies the question was not seen and-1 implies the } \\
\text { question was shown but not answered. }\end{array}$ \\
\hline
\end{tabular}

If either geoip_country or reported_country were not in the set of sampled countries, we report null for country. If self-reported country was present and in the sampled set, we infer country equal to the self-reported country. If a self-reported country was missing, or the self-reported country was not in the sampled set, we infer the country equal to the co untry from the GeolP field. If the GeolP country is missing or not in the sampled countries as well, then we do not infer a country and this field will be null. 


\section{Information Exposure}

- info_exposure_past_week (string): In the past week, how much, if anything, have you heard or read about coronavirus (COVID-19)? [Nothing, A little, A moderate amount, A lot]

- info_exposure_more_less_wanted (string): In the past week, did you see more or less news than you wanted to see about coronavirus (COVID-19)? [Much more, More, About the right amount, Less, Much less]

\section{Knowledge and Positive Cases}

- knowledge_existing_treatments (string): Which of the following is correct? [There is a drug to treat COVID-19, There is a vaccine for COVID-19, There is both a drug for treatment and a vaccine for COVID-19, There is currently no drug treatment or vaccine for COVID-19, I am unsure which is correct]

- know_positive_case (string): Do you personally know someone who has tested positive for COVID-19? [Yes, No, Prefer not to say]

\section{Vaccine and Healthcare}

- vaccine_accept (string): If a vaccine for COVID-19 becomes available, would you choose to get vaccinated? [Yes, No, Don't know, I have already been vaccinated]

- healthcare_avoid_contact (string): In the past week, have you avoided contact with health care facilities or health care workers due to fear of exposure to COVID-19? [Yes, No]

- healthcare_availability (string): In the past week, have you been able to get an appointment with a healthcare worker when you needed one, whether related to COVID19 or not? [Yes, No, I needed one and was not able, No, I did not need an appointment]

- Flu_vaccine: (string) Have you taken a flu vaccine this fall or do you plan to take one in the coming weeks? [Yes, No, Don't Know]. This question was only fielded in North American countries.

\section{Demographics}

- gender (string): [Male, Female, Other]

- age (string): [Under 20, 20-30, 31-40, 41-50, 51-60, 61-70, 71-80, Over 80]

- education (string): What is the highest level of education you have completed? [Less than primary school, Primary school, Secondary school, College / university, Graduate school]

- own_health (string): In general, how would you rate your overall health? [Excellent, Very good, Good, Fair, Poor]

- country (string): In which country do you currently reside?

- us_state (string): In which state do you currently reside?

- Only asked if country == United States of America

- india_state (string): In which state or union territory do you currently reside? 
- Only asked if country $==$ India

- density (string): Which of these best describes the area where you are currently staying? [City, Town, Village or rural area]

\section{Information Sources, Trust \& Needs}

- Information Medium: In the past week, from which of the following, if any, have you received news and information about COVID-19? Select all that apply. [1=option selected, $0=$ option shown but not selected, and another option was selected. Note: if all values have -1 or -2 , this question was viewed and the options were presented, but no options were selected.]

- news_medium_online_sources: Online sources (websites, apps, social media)

- news_medium_messaging_apps: Messaging apps / SMS/ text messaging

- news_medium_newspapers: Newspapers

- news_medium_television: Television

- news medium_radio: Radio

- Information Sources: In the past week, from which of the following, if any, have you received news and information about COVID-19? Select all that apply. [1=option selected, $0=$ option shown but not selected, and another option was selected. Note: if all values have -1 or -2 , this question was viewed and the options were presented, but no options were selected.]

- news_sources_local_health_workers: Local health workers, clinics, and community organizations

- news_sources_scientists: Scientists, doctors, and health experts

- news_sources_world_health_organization: World Health Organization (WHO)

- news_sources_government_health_authorities: Government health authorities or other officials

- news_sources_politicians: Politicians

- news_sources_journalists: Journalists

- news_sources_ordinary_people_i_know_personally: Ordinary people I know personally

- news_sources_ordinary_people_i_dont_know_personally: Ordinary people I don't know personally

- Information Medium Trust: How much do you trust each of the following as a source of COVID-19 news and information? [Do not trust, Somewhat trust, Trust]

- news_medium_trust_online_sources: Online sources (websites, apps, social media)

- news_medium_trust_messaging_apps: Messaging apps / SMS/ text messaging

- news_medium_trust_newspapers: Newspapers

- news_medium_trust_television: Television 
- news medium trust_radio: Radio

- Information Sources Trust: How much do you trust each of the following as a source of COVID-19 news and information? [Do not trust, Somewhat trust, Trust]

- news_sources_trust_local_health_workers: Local health workers, clinics, and community organizations

- news_sources_trust_scientists: Scientists, doctors, and health experts

o news_sources_trust_world_health_organization: World Health Organization (WHO)

- news_sources_trust_government_health_authorities: Government health authorities or other officials

- news_sources_trust_politicians: Politicians

- news_sources_trust_journalists: Journalists

- news_sources_trust_ordinary_people_i_know_personally: Ordinary people I know personally

o news_sources_trust_ordinary_people_i_do_not_know_personally: Ordinary people I don't know personally

- Information Demand: Which of the following aspects of COVID-19 do you have the most questions about? [ $1=$ option selected, $0=$ option shown but not selected, and another option was selected. Note: if all values have -1 or -2 , this question was viewed and the options were presented, but no options were selected.]

o information_demand_the_cause_of_the_disease: The cause of the disease

- Information_demand_sy mptoms_and_risk_factors: Symptoms and risk factors

- information_demand_treatment_of_the_disease: Treatment of the disease

o information_demand_how_i_can_protect_myself: How I can protect myself

- information_demand_immunity: Immunity

- information_demand_vaccine: Scientific progress in development of a vaccine or treatment

- information_demand_how_other_people_are_coping: How other people are coping

- information_demand_caring_for_those_most_at_risk_of_covid: Caring for those most at risk of COVID-19

- information_demand_education: How I can best take care of my children's school education

- information_demand_differences_between_covid_and_other_dise ases: Differences between COVID-19 and other diseases (e.g. flu)

- information_demand_the_evolution_of_the_pandemic_in: The evolution of the pandemic in [country selected]

o information_demand_the_evolution_of_the_pandemic_globally: The evolution of the pandemic globally 
- information_demand_the_economic_impact_of_covid_to_me_perso nally: The economic impact of COVID-19 to me personally

- information_demand_the_economic_impact_of_covid_in: The economic impact of COVID-19 in [country selected]

o information_demand_how_to_maintain_my_mental_health_during_ the isolation: How to maintain my mental health during the isolation

o information_demand_how_to_maintain_my_social_contact_despit e_the_physical_distancing: How to maintain my social contact despite the physical distancing

- information_demand_other_protection_measures_by_the_governm ent_and_communities: Other protection measures by the government and communities

\section{Basic Knowledge}

- Knowledge Risk Groups: Which of the following types of people are at the highest risk of severe illness from COVID-19? Select all that apply. [1=option selected, $0=0$ ption shown but not selected, and another option was selected. Note: if all values have -1 or -2 , this question was viewed and the options were presented, but no options were selected.]

o knowledge_high_risk_groups_people_of_certain_religions:

People of certain religions

o knowledge_high_risk_groups_people_of_certain_ages: People of certain ages

o knowledge_high_risk_groups_people_with_certain_medical_cond itions: People with certain medical conditions

o knowledge_high_risk_groups_people_with_certain_ethnic_backg rounds: People with certain ethnic backgrounds

o knowledge_high_risk_groups_none_of_the_above: None of the above

- knowledge_spread: Which of the following best describes how COVID-19 spreads? (string) [Human contact, coughing or sneezing, Other, Dirt or pollution, Exposure to animals, Mosquito bites]

- Knowledge Symptoms: Which of the following can be symptoms of COVID-19? Please select as many as apply. [ $1=$ option selected, $0=$ option shown but not selected, and another option was selected. Note: if all values have -1 or -2 , this question was viewed and the options were presented, but no options were selected.]

- knowledge_symptoms_fever: Fever

o knowledge_symptoms_cough: Cough

- knowledge_symptoms_shortness_of_breath: Shortness of breath

o knowledge_symptoms_sore_throat: Sore throat

- knowledge_symptoms_runny_or_stuffy_nose: Runny or stuffy nose

- knowledge_symptoms_muscle_or_body_aches: Muscle or body aches

- knowledge_symptoms_headaches: Headaches

- knowledge_symptoms_fatigue: Fatigue (tiredness) 
- knowledge_symptoms_diarrhea: Diarrhea

- knowledge_symptoms_loss_of_taste_and_smell: Loss of taste and smell

o knowledge_symptoms_none_of_these: None of these

\section{Distancing Familiarity, Importance \& Norms}

- distancing_familiarity (string): Which of the following best describes your familiarity with the term "physical distancing" during the COVID-19 pandemic? [I have not heard of it, I have heard of it but do not know what it means, I have heard of it and have some idea of what it means, I have heard of it and know what it means]

- distancing_importance (string): How important do you think physical distancing is for slowing the spread of COVID-19? [Not at all important, Slightly important, Moderately important, Very important, Extremely Important]

- This question was only shown to respondents with distancing_familiarity=l have heard of it and have some idea of what it means or distancing_familiarity=l have heard of it and know what it means

- Distancing Norms: Out of 100 people in your community, how many do you think do the following when they go out in public?

- distancing_norms_maintain_a_distance_of_at_least_1_meter_fr om_others: Maintain a distance of at least 1 meter from others

o distancing_norms_wear_a_face_mask_or_covering: Wear a face mask or covering

- norms_vaccine: Out of 100 people in your community, how many do you think would take a COVID-19 vaccine if it were made available?

\section{Risk Perceptions and Locus of Control}

- risk_community (string): How dangerous do you think the COVID-19 risk is to your community? [Not at all dangerous, Slightly dangerous, Moderately dangerous, Very dangerous, Extremely dangerous]

- risk_infection (string): How likely is it that someone of the same age as you in your community becomes sick from COVID-19? [Not at all likely, Slightly likely, Moderately likely, Very likely, Extremely likely]

- control_infection (string): Do you agree with this statement? "I have control over whether I will get COVID-19." [Strongly disagree, Somewhat disagree, Neither agree or disagree, Somewhat agree, Strongly agree]

- infection_severity (string): How serious would it be if you became infected with COVID-19? [Not at all serious, Somewhat serious, Very serious]

\section{Prevention Behaviors in Practice}

- prevention_distancing (string): How often are you able to stay at least 1 meter away from people not in your household? [Never, Rarely, Sometimes, Often, Always] 
- prevention_hand_washing (string): When you clean your hands, how often are you able to clean your hands with soap or alcohol-based handrub? [Never, Rarely, Sometimes, Often, Always]

- prevention_mask (string): How often are you able to wear a mask or face covering when you are in public? [Never, Rarely, Sometimes, Often, Always] This question was only added starting in wave 3 on 8/4/2020 at 2:15PM ET.

\section{Behavioral Measures Taken, Efficacy \& Norms}

- Preventative Measures Taken: What measures have you taken to prevent infection from COVID-19 in the past week? [ $1=$ option selected, $0=$ option shown but not selected, and another option was selected. Note: if all values have -1 or -2 , this question was viewed and the options were presented, but no options were selected.]

- measures_taken_washing_hands: Washing hands regularly using disinfectants or soap and water

- measures_taken_cover_coughs: Covering mouth and nose when coughing or sneezing

- measures_taken_avoid_sick: Avoiding close contact with anyone who has a fever and cough

- measures_taken_wearing_a_face_mask_or_covering: Wearing a face mask or covering

- measures_taken_meter_distance: Staying at least 1 meter away from other people when out in public

- measures_taken_avoid_touching_face: Avoiding touching your eyes, nose, and mouth with unwashed hands

- measures_taken_taking_herbal_supplements: Taking herbal supplements

- measures_taken_using_homeopathic_remedies: Using homeopathic remedies

- measures_taken_caution_opening_mail: Using caution when opening letters and packages

- measures_taken_getting_the_flu_vaccine: Getting the flu vaccine

- measures_taken_eating_garlic: Eating garlic, ginger, or lemon

- measures_taken_cleaning_or_disinfecting_surfaces: Cleaning or disinfecting surfaces

- measures_taken_using_antibiotics: Using antibiotics

- measures_taken_cleaning_or_disinfecting_a_mobile_phone:

Cleaning or disinfecting a mobile phone

- measures_taken_isolation: Self-isolation

- measures_taken_none_of_these: None of these

- effect_hand_washing (string): How effective is handwashing for preventing the spread of COVID-19? [Extremely effective, Very effective, Moderately effective, Slightly effective, Not effective at all] 
- effect_mask (string): How effective is wearing a face mask for preventing the spread of COVID-19? [Extremely effective, Very effective, Moderately effective, Slightly effective, Not effective at all]

- country_management (string): How well is COVID-19 being handled in [country selected]? [Extremely well, Very well, Moderately well, Slightly well, Not well at all]

- community_management (string): How well is your community handling COVID-19? [Extremely well, Very well, Moderately well, Slightly well, Not well at all]

- community_action_importance (string): How important is it for you to take actions to prevent the spread of COVID-19 in your community? [Extremely important, Very important, Moderately important, Slightly important, Not important at all]

- community_action_norms (string): How important do other people in your community think it is to take actions to prevent the spread of COVID-19? [Extremely important, Very important, Moderately important, Slightly important, Not important at all]

- Community Norms: Out of 100 people in your community, how many do you think believe the following because of COVID-19? (Default slider at 50)

- community_norms_social_gatherings_should_be_cancelled: Social gatherings should be cancelled

- community_norms_maintain_1_meter: People should stay at least 1 meter away from other people when out in public

- community_norms_mask: People should wear a face mask or covering when out in public

- community_norms_close_retail: Non-essential retail shops should be closed

- community_norms_a_general_curfew_should_be_enforced: A general curfew should be enforced

- community_norms_travel_between_countries_should_be_restrict ed: Travel between countries should be restricted

- community_norms_travel_within_the_country_should_be_restric ted: Travel within the country should be restricted

\section{Employment and Work}

- employed_2020 (string): Have you been working at some point in 2020? [Yes, No]

- work_changes (string): How has your work changed since January 31, 2020? [No longer employed, Newly employed, Employed in a different business, Role substantially changed with same business, Little change]

- work_type (string): Which best way to describe the work you do most of the time to make money? [I work for my own business, I work in a business that is run by my household or family member, I work in a business that is run by someone else, I work for the government, Other]

- work_industry (string): What is the main activity of the business or organization in which you were working before February 2020? [Agriculture, Buying and selling, Construction, Education, Electricity/water/gas/waste, Financial/insurance/real estate 
services, Health, Manufacturing, Mining, Personal services,

Professional/scientific/technical activities, Public administration,Tourism, Transportation, Other]

\section{Intentions to Visit Locations}

- Open Locations: Which of the following businesses, locations, or events are currently open and operating near you? [ $1=$ option selected, $0=$ option shown but not selected, and another option was selected. Note: if all values have -1 or -2 , this question was viewed and the options were presented, but no options were selected.]

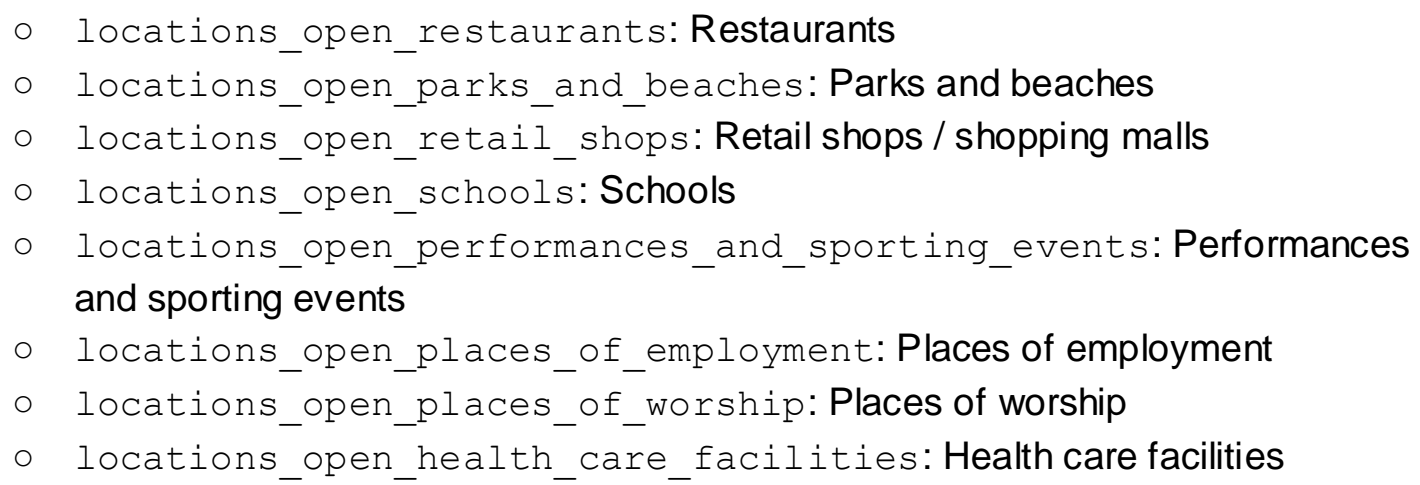

- Locations Would Attend: Which of the following businesses, locations, or events would you visit or attend in the coming two weeks if they were operating at full capacity? [ $1=$ option selected, $0=$ option shown but not selected, and another option was selected. Note: if all values have -1 or -2 , this question was viewed and the options were presented, but no options were selected.]

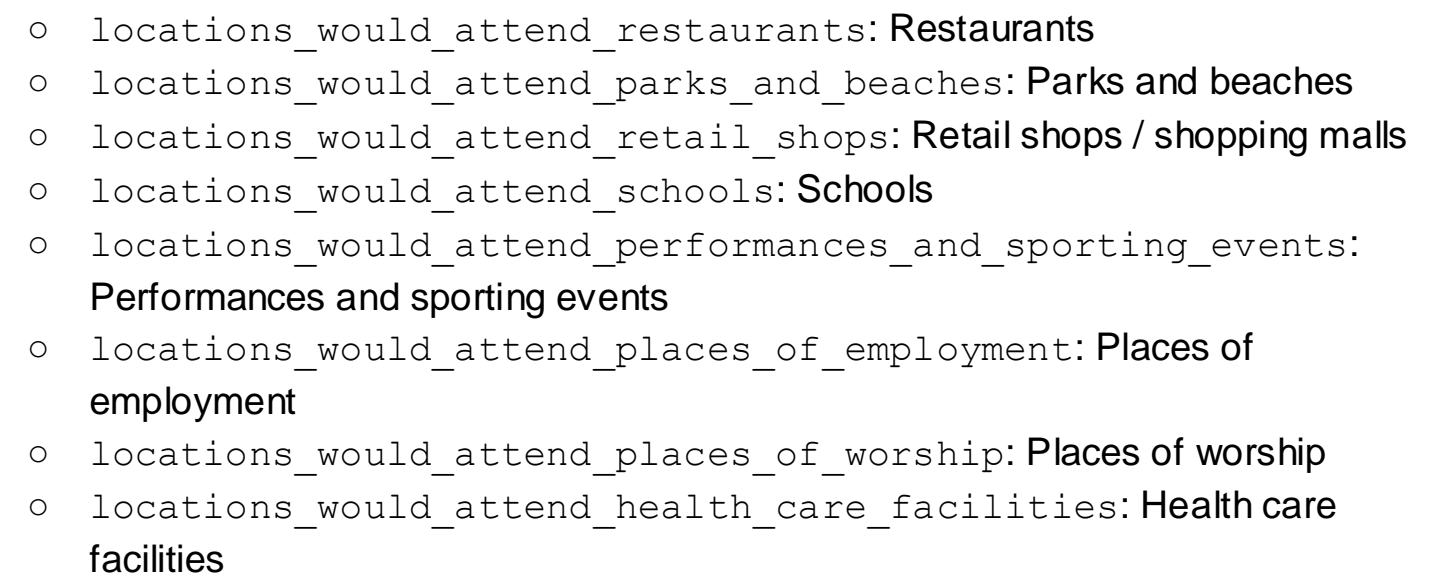

- Preventative Measures: Compared to a [location] with no precautions at all, are you more or less likely to visit a restaurant with the following precautions? [More likely to visit, Doesn't affect my actions, Less likely to visit]

- Asked for the following locations: restaurant, healthcare facility, place of worship, and retail shop (location could be restaurants, worship, health or retail)

- prevent_measures_[location]_operating_at_limited_capacity: Operating at limited capacity 
- prevent_measures_[location]_everyone_has_to_wear_a_mask:

Everyone has to wear a mask

- prevent_measures_[location]_everyone_has_to_pass_a_temperat

ure_check: Everyone has to pass a temperature check

- prevent_measures_[location]_has_additional_hand_washing_sta tions: Has additional hand washing stations

\section{Travel}

- travel_restrictions (string): Have you refrained from traveling between cities because of restrictions on movement due to COVID-19? [Yes, No - I planned to travel and kept plans, No - I did not plan to travel]

- travel_concerns (string): Have you refrained from traveling between cities due to fears regarding COVID-19? [Yes, No - I planned to travel and kept plans, No - I did not plan to travel]

\section{Future Actions}

- future_masks (string): Over the next two weeks, how likely are you to wear a mask when in public? [Always, Almost always, When convenient, Rarely, Never]

- future_distancing (string): Over the next two weeks, how likely are you to maintain a distance of at least 1 meter from others when in public? [Always, Almost always, When convenient, Rarely, Never]

- future_vaccine (string): If a vaccine against COVID-19 infection is available in the market, would you take it? [Yes, definitely; Probably; Unsure; Probably not; No, definitely not]

- Only shown if vaccine_accept != "I have already been vaccinated"]

- Would you be more or less likely to take a vaccine against COVID-19 infection if it were made available and recommended to you by each of the following [More likely, Less likely, No impact]

- future_vaccine_friends_and_family (string): Friends and family

- future_vaccine_local_health_workers (string): Local health workers

- future_vaccine_world_health_organization (string): WHO

- future_vaccine_government_health_officials (string): Government health officials

○ future_vaccine_politicians (string): Politicians

\section{Survey Information}

Starting in wave 9 of the survey, we added an information block that shared an example of the information that was being learned from the survey with respondents. The information was the (weighted) average number of respondents saying they practice (or would accept for vaccines) a preventative behavior. Respondents self reporting they were in a wave country were randomly shown one of three behaviors (vaccines, masks, or distancing) and were told 
Your responses to this survey are helping researchers in your region and around the world understand how people are responding to COVID-19. For example, we estimate from survey responses in the previous month that [[country share]]\% of people in your country say they [[broad or narrow]] [[preventative behavior]].

The blanks were filled in with one randomly chosen preventative behavior, a broad or narrow definition of the activity, and the true share of responses for the respondent's country. The three behaviors were vaccine acceptance, mask wearing, and social distancing. In the broad condition, we used a more inclusive definition of the preventative behavior and the narrow condition used a more restrictive definition. For example, for vaccine acceptance we either reported the share of people responding "Yes" or the share of people responding "Yes" or "Don't know" to the baseline vaccine acceptance question.

- eligible_for_information (int): An indicator if the respondent was eligible for the information. This includes all individuals with survey_information_provided $==1$ and additional individuals who dropped off before the end of the tracking questions.

- survey_information_provided (int): An indicator if the information was provided, or the information was going to be provided but the respondent stopped the survey before reaching the information block (but finished the tracking questions).

- survey_information_behavior (string): A string indicating the behavior the information pertained to, if provided. One of vaccine, maks, dist.

- survey_information_level (string): A string indicating whether the respondent was shown the broad (high) definition or the narrow (low) definition of compliance. 


\section{Appendix B: Survey Change Log}

- 2020-07-12: Changed the intentions to visit with precautions randomization to randomly sample two locations rather than one

- 2020-07-25: Added Taiwan as a country option

- 2020-07-27: Added Republic of Northern Macedonia as a country option

- 2020-07-28: Shortened allowed survey response time to record partial completes 4 hours after latest activity from 48 hours

- 2020-08-04: Added an additional mask question (How often are you able to wear a mask or face covering when you are in public?)

- 2020-09-14: Added race question for the US (Not yet released to partners given as we work on preserving privacy)

- 2020-09-14: Changed randomization so that the BEN and Prevention Behaviors in Practice blocks are sampled for all users. Added one more block to be sampled so other blocks are sampled at the same rate.

- 2020-10-28: Survey V2 launched

- Added future behavior block

- Added vaccine norm question

- Added Flu question

- Removed healthcare access question (replaced w/ flu)

- Removed past travel block

- Removed distancing familiarity question

- Removed intentions to visit if open question

- Removed locations open questions

- 2021-01-18: Added option "I have already been vaccinated" to vaccine_accept question. 
Appendix C: Survey Instrument

\section{Survey Flow}




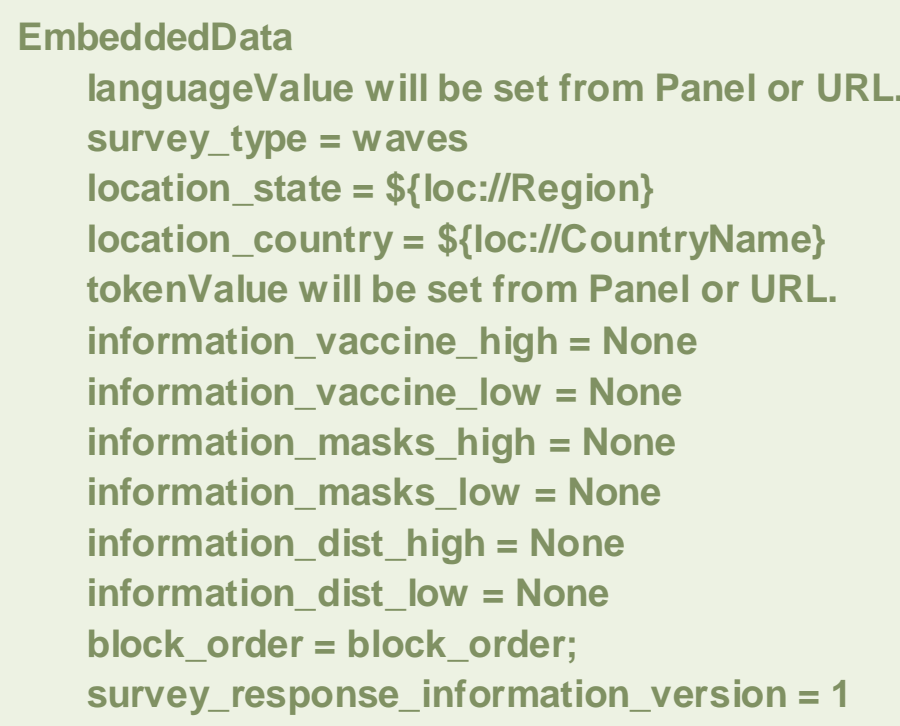

Block: Briefing (7 Questions)

Branch: New Branch

If

If Are you 18 years or older? No Is Selected

Or Do you consent to take part in this survey run by the Massachusetts Institute of Technology? No Is Selected

Or Do you consent to sharing the information with external researchers? No Is

Selected

Block: Debrief - Terminated (2 Questions)

\section{EndSurvey:}

Standard: COVID-19 information exposure (3 Questions)

Block: Knowledge and positive cases (4 Questions)

Standard: Vaccine and healthcare (4 Questions)

Standard: Demographics (16 Questions)

BlockRandomizer: 6 -

Group: BEN

Standard: Behavioral measures taken (2 Questions)

Standard: Beliefs about efficacy (6 Questions)

Standard: Importance and norms (5 Questions)

Standard: Prevention behaviors in practice (5 Questions)

Standard: FutureActions (5 Questions)

Standard: Distancing familiarity, importance \& norms (5 Questions)

Branch: New Branch

If 
If information_vaccine_high Is Not Equal to None And information_vaccine_low Is Not Equal to None And information_masks_high Is Not Equal to None And information_masks_low Is Not Equal to None And information_dist_high Is Not Equal to None And information_dist_low Is Not Equal to None

Standard: SurveyResponselnformation (7 Questions)

BlockRandomizer: 2 -

Group: Information Block

Standard: Information sources (3 Questions)

Standard: Information sources - Trust (3 Questions)

Standard: Information Needs (2 Questions)

Standard: Basic knowledge (6 Questions)

Standard: Risk perceptions and locus of control (6 Questions)

Standard: Work (5 Questions)

Group: Intentions to visit

Standard: Intentions to visit locations if open (2 Questions)

Block: Debrief (2 Questions)

\section{EndSurvey: Default}


Start of Block: Briefing

Q1.1 Browser Meta Info

Browser (1)

Version (2)

Operating System (3)

Screen Resolution (4)

Flash Version (5)

Java Support (6)

User Agent (7)

\section{Display This Question:}

If GeolP Location CountryCode != US

Q1.2 About This Survey The goal of this survey is to gain a better public understanding of knowledge, attitudes and practices related to COVID-19 across the world. It is part of a research study led by the Massachusetts Institute of Technology (MIT). You may stop taking the survey at any time and may skip any question. This survey is voluntary and will take about 7

minutes. How Your Information Will Be Used

\section{Information We Collect}

The information shall be processed for this research purpose only.

This study is conducted by MIT, not by Facebook. The only information MIT receives about you from Facebook is your language preference, a randomly assigned ID number and a statistical number. Facebook generates the statistical number based on information such as your age, gender, location and other information. This statistical number we receive is a single number that will not reveal any specific information about you, it simply helps us with our statistical analysis, to ensure that the information taken from these surveys is representative for your country.

In order to ensure that MIT cannot identify you and to keep your responses confidential, MIT does not collect your name, email or any other information that could be used to identify you. Your responses will be retained indefinitely. Information We Share No individual responses will be shared back to Facebook. Any research results shared with the public will be aggregated and will not identify you individually or your

\section{responses. Agreements}

You must be 18 years or older to take this survey.

If you have questions about the study or on the use of your information, please contact: covid-survey@lists.mit.edu. For data protection information, please see the section "Rights for Individuals in the European Economic Area" on the MIT Data Policy, which provides the relevant further information on your rights if/where personal data is processed as part of this research project in accordance with applicable data protection law. 
For this research to be effective, we want to share your responses, along with your randomly assigned ID number and your statistical number, with external researchers in your country and elsewhere. These researchers will include academic institutions and non-governmental organizations. This will include researchers at institutions, such as Johns Hopkins University and the World Health Organization. They follow the applicable security and data protection safeguards and will only use the stated information for the research we have described and will not use the information to identify you.

Display This Question:

If GeolP Location CountryCode = US

Q1.3 About This Survey The goal of this survey is to gain a better public understanding of knowledge, attitudes and practices related to COVID-19 across the world. It is part of a research study led by the Massachusetts Institute of Technology (MIT). You may stop taking the survey at any time and may skip any question. This survey is voluntary and will take about 7

minutes. How Your Information Will Be Used Information We Collect

The information shall be processed for this research purpose only.

This study is conducted by MIT, not by Facebook. The only information MIT receives about you from Facebook is your language preference, a randomly assigned ID number and a statistical number. Facebook generates the statistical number based on information such as your age, gender, location and other information. This statistical number we receive is a single number that will not reveal any specific information about you, it simply helps us with our statistical analysis, to ensure that the information taken from these surveys is representative for your country.

In order to ensure that MIT cannot identify you and to keep your responses confidential, MIT does not collect your name, email or any other information that could be used to identify you.

Your responses will be retained indefinitely.

\section{Information We Share}

No individual responses will be shared back to Facebook. Any research results shared with the public will be aggregated and will not identify you individually or your responses.

\section{Agreements}

You must be 18 years or older to take this survey.

If you have questions about the study or on the use of your information, please contact: covid-survey@lists.mit.edu.

For this research to be effective, we want to share your responses, along with your randomly assigned ID number and your statistical number, with external researchers in your country and elsewhere. These researchers will include academic institutions and non-governmental organizations. This will include researchers at institutions, such as Johns Hopkins University and the World Health Organization. They follow the applicable security and data protection 
safeguards and will only use the stated information for the research we have described and will not use the information to identify you.

Q1.4 Are you 18 years or older?

Yes (1)

No (2)

Q1.5 Do you consent to take part in this survey run by the Massachusetts Institute of Technology?
Yes (1)
No (2)

Q1.6 Do you consent to sharing the information with external researchers?
Yes (1)
No (2)

Q1.7 Timing

First Click (1)

Last Click (2)

Page Submit (3)

Click Count (4) 


\section{End of Block: Briefing}

Start of Block: Debrief - Terminated

Q2.1

Thank you for considering participating in this survey.

According to the World Health Organization (WHO), Coronavirus (COVID-19) is an infectious disease where older people are more likely to develop serious illness.

The best way to prevent the spread is through techniques like: Washing your hands regularly with soap and water Covering your mouth and nose when coughing or sneezing Maintaining at least 1 meter distance between you and other people Wearing a face mask or covering when in public or unable to keep distance from others

You can find the WHO's guidance on Coronavirus (COVID-19) here.

Q2.2 Timing

First Click (1)

Last Click (2)

Page Submit (3)

Click Count (4)

\section{End of Block: Debrief = Terminated}

Start of Block: COVID-19 information exposure

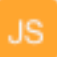

Q3.1 Timing

First Click (1)

Last Click (2)

Page Submit (3)

Click Count (4) 
Q3.2 In the past week, how much, if anything, have you heard or read about coronavirus (COVID-19)?

Nothing (1)

A little (2)

A moderate amount (3)

A lot (4)

Q3.3 In the past week, did you see more or less news than you wanted to see about coronavirus (COVID-19)?

Much more (1)

More (2)

About the right amount (3)

Less (4)

Much less (5)

\section{End of Block: COVID-19 information exposure}

Start of Block: Knowledge and positive cases

JS

Q4.1 Timing

First Click (1)

Last Click (2)

Page Submit (3)

Click Count (4) 
Q4.2 Which of the following is correct?

There is a drug to treat COVID-19 (1)

There is a vaccine for COVID-19 (2)

There is both a drug for treatment and a vaccine for COVID-19 (3)

There is currently no drug treatment or vaccine for COVID-19 (4)

I am unsure which is correct (5) 
Q4.3 Timing

First Click (1)

Last Click (2)

Page Submit (3)

Click Count (4)

Q4.4 Do you perso nally know someone who has tested positive for COVID-19?

Yes (1)

No (2)

Prefer not to say (3) 


\section{End of Block: Knowledge and positive cases}

Start of Block: Vaccine and healthcare

Q5.1 Timing

First Click (1)

Last Click (2)

Page Submit (3)

Click Count (4)

Q5.2 If a vaccine for COVID-19 becomes available, would you choose to get vaccinated?

Yes (1)

No (2)

Don't know (3) 
Q5.3 In the past week, have you avoided contact with health care facilities or health care workers due to fear of exposure to COVID-19?
Yes (1)
No (2)

\section{Display This Question:}

If GeolP Location CountryCode $!=I D$

And GeolP Location CountryCode $!=A R$

And GeolP Location CountryCode $!=B R$

Q5.6 Have you taken a flu vaccine this fall or do you plan to take one in the coming weeks?

Yes (1)

No (2)

Don't know (3)

\section{End of Block: Vaccine and healthcare}

\section{Start of Block: Demographics}

Q6.1 Timing

First Click (1)

Last Click (2)

Page Submit (3)

Click Count (4) 
Q6.2 What is your gender?

Male (1)

Female (2)

Other (3)

Q6.3 What is your age?

Under 20 (1)

20-30 (2)

$31-40 \quad(3)$

$41-50(4)$

51-60 (5)

61-70 (6)

$71-80$ (7)

Over 80 (8) 
Q6.4 Timing

First Click (1)

Last Click (2)

Page Submit (3)

Click Count (4)

Page Break 
Q6.5 Timing

First Click (1)

Last Click (2)

Page Submit (3)

Click Count (4)

Q6.6 What is the highest level of education you have completed?

Less than primary school (1)

Primary school (5)

Secondary school (2)

College / university (3)

Graduate school (4) 
Q6.7 Timing

First Click (1)

Last Click (2)

Page Submit (3)

Click Count (4)

Q6.8 In general, how would you rate your overall health?
Excellent (1)
Very good
(2)
Good (3)
Fair (4)
Poor (5) 
Q6.9 Timing

First Click (1)

Last Click (2)

Page Submit (3)

Click Count (4)

$X \rightarrow$

Q6.10 In which country do you currently reside?

Afghanistan (1) ... Zimbabwe (1357)

Display This Question:

If List of Countries = United States of America

Q6.11 In which state do you currently reside?

Alabama (1) ... I do not reside in the United States (53)

Display This Question:

If List of Countries = India

Q6.12 In which state or union territory do you currently reside?

Andaman and Nicobar Islands (1) ... West Bengal (36) 
Q6.13 Timing

First Click (1)

Last Click (2)

Page Submit (3)

Click Count (4)

JS

Q6.14 Which of these best describes the area where you are currently staying?

City (1)

Town (2)

Village or rural area (3) 
Q6.15 Are you of Hispanic, Latino, or Spanish origin?

Yes (1)

No, not of Hispanic, Latino, or Spanish origin (2)

Q6.16 What is your race?

American Indian or Alaska Native (1)

Asian (2)

Black or African American (3)

Native Hawaiian or other Pacific Islander (4)

White (5)

Some other race (6)

\section{End of Block: Demographics}

Start of Block: Behavioral measures taken

Q14.1 Timing

First Click (1)

Last Click (2)

Page Submit (3)

Click Count (4) 
JS $y_{4}$ 
Q14.2 What measures have you taken to prevent infection from COVID-19 in the past week?

Washing hands regularly using disinfectants or soap and water (1)

Covering mouth and nose when coughing or sneezing (2)

Avoiding close contact with anyone who has a fever and cough (3)

Wearing a face mask or covering (4)

Staying at least 1 meter away from other people when out in public (5)

Staying at least 2 meters away from other people when out in public (6)

Avoiding touching your eyes, nose, and mouth with unwashed hands (7)

Taking herbal supplements (8)

Using homeopathic remedies (17)

Using caution when opening letters and packages (9)

Getting the flu vaccine (10)

Eating garlic, ginger, or lemon (11)

Cleaning or disinfecting surfaces (12)

Using antibiotics (13)

Cleaning or disinfecting a mobile phone (14)

Self-isolation (15)

$\bigotimes$ None of these (16) 
Page Break 


\section{End of Block: Behavioral measures taken}

Start of Block: Beliefs about efficacy

Q15.1 Timing

First Click (1)

Last Click (2)

Page Submit (3)

Click Count (4)

Q15.2 How effective is handwashing for preventing the spread of COVID-19?

Extremely effective (1)

Very effective (2)

Moderately effective (3)

Slightly effective (4)

Not effective at all (5)

Q15.3 How effective is wearing a face mask for preventing the spread of COVID-19?

Extremely effective (1)

Very effective (2)

Moderately effective (3)

Slightly effective (4)

Not effective at all (5) 
Q15.4 Timing

First Click (1)

Last Click (2)

Page Submit (3)

Click Count (4)

Q15.5 How well is COVID-19 being handled in $\$\{$ Q6.10/ChoiceGroup/SelectedChoices $\}$ ?

Extremely well (1)

Very well (2)

Moderately well (3)

Slightly well (4)

Not well at all (5)

Q15.6 How well is your community handling COVID-19?

Extremely well (1)

Very well (2)

Moderately well (3)

Slightly well (4)

Not well at all (5)

\section{End of Block: Beliefs about efficacy}


Q16.1 Timing

First Click (1)

Last Click (2)

Page Submit (3)

Click Count (4)

Q16.2 How important is it for you to take actions to prevent the spread of COVID-19 in your community?
Extremely important (1)
Very important (2)
Moderately important (3)
Slightly important (4)
Not at all important (5)

Q16.3 How important do other people in your community think it is to take actions to prevent the spread of COVID-19?

Extremely important (1)

Very important (2)

Moderately important (3)

Slightly important (4)

Not at all important (5) 
Q16.4 Timing

First Click (1)

Last Click (2)

Page Submit (3)

Click Count (4)

Q16.5 Out of 100 people in your community, how many do you think believe the following because of COVID-19?

\section{0}

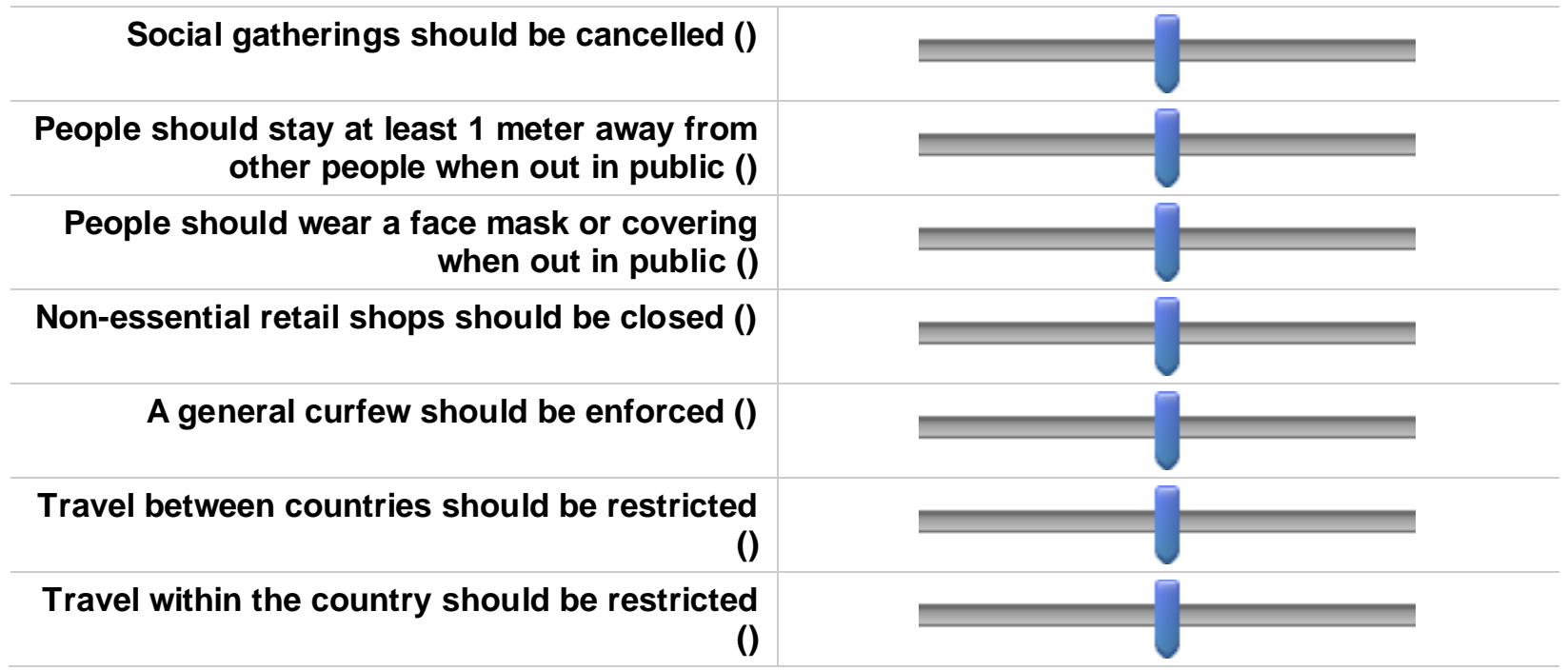




\section{End of Block: Importance and norms}

\section{Start of Block: Prevention behaviors in practice}

Q13.1 Timing

First Click (1)

Last Click (2)

Page Submit (3)

Click Count (4)

Q13.2 How often are you able to stay at least 1 meter away from people not in your household?

Never (1)

Rarely (2)

Sometimes (3)

Often (4)

Always (5) 
Q13.3 Timing

First Click (1)

Last Click (2)

Page Submit (3)

Click Count (4)

Q13.4 When you clean your hands, how often are you able to clean your hands with soap or alcohol-based handrub?

Never (1)

Rarely (2)

Sometimes (3)

Often (4)

Always (5)

Q13.5 How often are you able to wear a mask or face covering when you are in public?

Never (1)

Rarely (4)

Sometimes (2)

Often (3)

Always (5) 
Q21.1 Timing

First Click (1)

Last Click (2)

Page Submit (3)

Click Count (4)

Q21.2 Over the next two weeks, how likely are you to wear a mask when in public?
Always (1)
Almost always (2)
When convenient (3)
Rarely (4)
Never (5)

Q21.3 Over the next two weeks, how likely are you to maintain a distance of at least 1 meter from others when in public?

Always (1)

Almost always (2)

When convenient (3)

Rarely (4)

Never (5) 
Q21.4 If a vaccine against COVID-19 infection is available in the market, wo uld you take it?
Yes, definitely
Probably (2)
Unsure (3)
Probably not (4)
No, definitely not (5)

Q21.5 Would you be more or less likely to take a vaccine against COVID-19 infection if it were made available and recommended to you by each of the following
More Likely (1)
Less Likely (2)
No Impact (3)

Friends and family (1)

Local health workers

(2)

World Health

Organization (WHO) (3)

Government health

officials (4)

Politicians (6) 
Q11.3 Timing

First Click (1)

Last Click (2)

Page Submit (3)

Click Count (4)

Q11.4 How important do you think physical distancing is for slowing the spread of COVID-19?

Not at all important (1)

Slightly important (2)

Moderately important (3)

Very important (4)

Extremely Important (5) 
Q11.5 Timing

First Click (1)

Last Click (2)

Page Submit (3)

Click Count (4)

Q11.6 Out of 100 people in your community, how many do you think do the following when they go out in public?

0102030405060708090100

\section{Maintain a distance of at least 1 meter from others ()}

Wear a face mask or covering ()

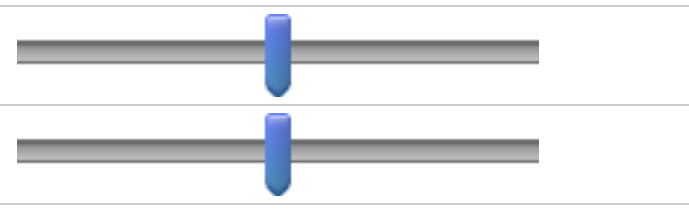

Q11.7 Out of 100 people in your community, how many do you think would take a COVID-19 vaccine if it were made available?

0102030405060708090100

\section{1()}

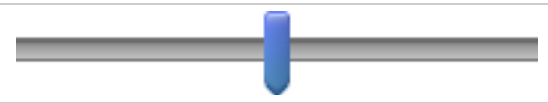

\section{End of Block: Distancing familiarity, importance \& norms}

\section{Start of Block: SurveyResponselnformation}

Q22.1 Timing

First Click (1)

Last Click (2)

Page Submit (3)

Click Count (4) 
Q22.2 Your responses to this survey are helping researchers in your region and around the world understand how people are responding to COVID-19. For example, we estimate from survey responses in the previous month that $\$\{$ e://Field/information_masks_high $\} \%$ of people in your country say they always or often wear a mask when in public.

JS

Q22.3 Your responses to this survey are helping researchers in your region and around the world understand how people are responding to COVID-19. For example, we estimate from survey responses in the previous month that $\$\{$ e://Field/information_masks_low $\} \%$ of people in your country say they always wear a mask when in public.

JS

Q22.4

Your responses to this survey are helping researchers in your region and around the world understand how people are responding to COVID-19. For example, we estimate from survey responses in the previous month that $\$\{$ e://Field/information_dist_high\} \% of people in your country say they are always or often able to maintain a distance of 1 meter when in public.

JS

Q22.5

Your responses to this survey are helping researchers in your region and around the world understand how people are responding to COVID-19. For example, we estimate from survey responses in the previous month that $\$\{$ e://Field/information_dist_low $\} \%$ of people in your country say they are always able to maintain a distance of 1 meter when in public.

JS

Q22.6

Your responses to this survey are helping researchers in your region and around the world understand how people are responding to COVID-19. For example, we estimate from survey responses in the previous month that $\$\{$ e://Field/information_vaccine_high $\} \%$ of people in your country say they may take a vaccine if one is made available. 
Q22.7

Your responses to this survey are helping researchers in your region and around the world understand how people are responding to COVID-19. For example, we estimate from survey responses in the previous month that $\$\{$ e://Field/information_vaccine_low $\} \%$ of people in your country say they will take a vaccine if one is made available. 


\section{End of Block: SurveyResponselnformation}

\section{Start of Block: Information sources}

Q7.1 Timing

First Click (1)

Last Click (2)

Page Submit (3)

Click Count (4)

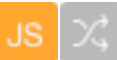

Q7.2 In the past week, from which of the following, if any, have you received news and information about COVID-19? Select all that apply.

Online sources (websites, apps, social media) (1)

Messaging apps / SMS / text messaging (2)

Newspapers (3)

Television (4)

Radio (5) 
Q7.3 In the past week, from which of the following, if any, have you received news and information about COVID-19? Select all that apply.

Local health workers, clinics, and community organizations (1)

Scientists, doctors, and health experts (2)

World Health Organization (WHO) (3)

Government health authorities or other officials (4)

Politicians (5)

Journalists (8)

Ordinary people I know personally (6)

Ordinary people I don't know personally (7) 
Start of Block: Information sources - Trust

Q8.1 Timing

First Click (1)

Last Click (2)

Page Submit (3)

Click Count (4)

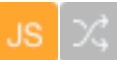

Q8.2 How much do you trust each of the following as a source of COVID-19 news and information?

\begin{tabular}{c|ccc} 
& Do not trust (1) & Somewhat trust (2) & Trust (3) \\
\hline $\begin{array}{c}\text { Online sources } \\
\text { (websites, apps, social } \\
\text { media) (1) }\end{array}$ & 0 & $\bigcirc$ \\
Messaging apps / SMS \\
/ text messaging (3) \\
Television (5)
\end{tabular}

Q8.3 How much do you trust each of the following as a source of COVID-19 news and information?

\begin{tabular}{c|ccr} 
& Do not trust (1) & Somewhat trust (2) & Trust (3) \\
\hline $\begin{array}{c}\text { Local health workers, } \\
\text { clinics, and community } \\
\text { organizations (1) }\end{array}$ & $\bigcirc$ & &
\end{tabular}


Scientists, doctors, health experts (31)

World Health Organization (WHO) (3)

Government health authorities or other officials (5)

Politicians (6)

Journalists (33)

Ordinary people I know personally (7)

Ordinary people I do not know personally

(32) 


\section{End of Block: Information sources - Trust}

Start of Block: Information Needs

Q9.1 Timing

First Click (1)

Last Click (2)

Page Submit (3)

Click Count (4) 
Q9.2 Which of the following aspects of COVID-19 do you have the most questions about?

The cause of the disease (1)

Symptoms and risk factors (2)

Treatment of the disease (3)

How I can protect myself (4)

Immunity (5)

Scientific progress in development of a vaccine or treatment (6)

How other people are coping (7)

Caring for those most at risk of COVID-19 (8)

How I can best take care of my children's school education (9)

Differences between COVID-19 and other diseases (e.g. flu) (10)

The evolution of the pandemic in \$\{Q6.10/ChoiceGroup/SelectedChoices $\}$ (11)

The evolution of the pandemic globally (12)

The economic impact of COVID-19 to me personally (13)

The economic impact of COVID-19 in \$\{6.10/ChoiceGroup/SelectedChoices\}

(14)

How to maintain my mental health during the isolation (15)

How to maintain my social contact despite the physical distancing (16)

Other protection measures by the government and communities (17) 


\section{End of Block: Information Needs}

\section{Start of Block: Basic knowledge}

Q10.1 Timing

First Click (1)

Last Click (2)

Page Submit (3)

Click Count (4)

Q10.2 Which of the following types of people are at the highest risk of severe illness from COVID-19? Select all that apply.

People of certain religions (1)

People of certain ages (2)

People with certain medical conditions (3)

People with certain ethnic backgrounds (4)

$\bigotimes$ None of the above (5) 
Q10.3 Timing

First Click (1)

Last Click (2)

Page Submit (3)

Click Count (4)

Q10.4 Which of the following best describes how COVID-19 spreads?

Human contact, coughing or sneezing (1)

Exposure to animals (2)

Mosquito bites (3)

Dirt or pollution (4)

Other (5) 
Q10.5 Timing

First Click (1)

Last Click (2)

Page Submit (3)

Click Count (4)

Q10.6 Which of the following can be symptoms of COVID-19? Please select as many as apply.

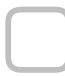

Fever (1)

Cough (2)

Shortness of breath (3)

Sore throat (4)

Runny or stuffy nose (5)

Muscle or body aches (6)

Headaches (7)

Fatigue (tiredness) (8)

Diarrhea (9)

Loss of taste and smell (10)

QNone of these (11) 


\section{End of Block: Basic knowledge}

Start of Block: Risk perceptions and locus of control

Q12.1 Timing

First Click (1)

Last Click (2)

Page Submit (3)

Click Count (4)

Q12.2 How dangerous do you think the COVID-19 risk is to your community?

Not at all dangerous (1)

Slightly dangerous (2)

Moderately dangerous (3)

Very dangerous (4)

Extremely dangerous (5) 
Q12.3 Timing

First Click (1)

Last Click (2)

Page Submit (3)

Click Count (4)

Q12.4 How likely is it that someone of the same age as you in your community becomes sick from COVID-19?
Not at all likely (1)
Slightly likely (2)
Moderately likely (3)
Very likely (4)
Extremely likely (5)

Q12.5 Do you agree with this statement? "I have control over whether I will get COVID-19."

Strongly disagree (1)

Somewhat disagree (4)

Neither agree or disagree (5)

Somewhat agree (6)

Strongly agree (7) 
Q12.6 How serious would it be if you became infected with COVID-19?

Not at all serious (1)

Somewhat serious (2)

Very serious (3) 


\section{Start of Block: Work}

Q17.1 Timing

First Click (1)

Last Click (2)

Page Submit (3)

Click Count (4)

Q17.2 Have you been working at some point in $2020 ?$

Yes (1)

No (2)

Q17.3 How has your work changed since January 31, 2020?

No longer employed (1)

Newly employed (2)

Employed in a different business (3)

Role substantially changed with same business (4)

Little change (5) 
Q17.4 Which best way to describe the work you do most of the time to make money?

I work for my own business (1)

I work in a business that is run by my household or family member (2)

I work in a business that is run by someone else (3)

I work for the government (4)

Other (5)

Display This Question:

If Have you been working at some point in 2020? = Yes 
Q17.5 What is the main activity of the business or organization in which you were working before February 2020?

Agriculture (1)

Buying and selling (11)

Construction (12)

Education (13)

Electricity/water/gas/waste (14)

Financial/insurance/real estate services (15)

Health (16)

Manufacturing (17)

Mining (18)

Personal services (19)

Professional/scientific/technical activities (20)

Public administration (21)

Tourism (22)

Transportation (23)

Other (24) 


\section{End of Block: Work}

\section{Start of Block: Intentions to visit locations if open}

Q18.1 Timing

First Click (1)

Last Click (2)

Page Submit (3)

Click Count (4)

Q18.4 Which of the following businesses, locations, or events would you visit or attend in the coming two weeks if they were operating at full capacity?

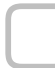

\section{Restaurants (1)}

Parks and beaches (2)

Retail shops / shopping malls (3)

Schools (4)

Performances and sporting events (5)

Places of employment (6)

Places of worship (7)

Health care facilities (8) 
Start of Block: Debrief

Q21.1 Timing

First Click (1)

Last Click (2)

Page Submit (3)

Click Count (4)

Q21.2

Thank you for participating in this survey, your answers will help researchers in your region and around the world understand how people are responding to COVID-19.

According to the World Health Organization (WHO), Coronavirus (COVID-19) is an infectious disease where older people are more likely to develop serious illness.

The best way to prevent the spread is through techniques like: Washing your hands regularly with soap and water Covering your mouth and nose when coughing or sneezing Maintaining at least 1 meter distance between you and other people Wearing a face mask or covering when in public or unable to keep distance from others

You can find the WHO's guidance on Coronavirus (COVID-19) here. 